\title{
Effect of turbulence intensity of fluid medium interface layer on magnetic liquid seal in liquid environment
}

\section{Wangxu Li}

Xihua University

Zhenggui Li ( $\sim$ Izhgui@mail.xhu.edu.cn )

Xihua University

Jie Cheng

Xihua University

Lianchen Xu

Xihua University

Xinrui Li

Xihua University

\section{Original Article}

Keywords: Liquid environment, magnetic liquid seal, numerical simulation, interface layer, turbulent intensity

Posted Date: April 8th, 2021

DOl: https://doi.org/10.21203/rs.3.rs-339189/v1

License: (c) (i) This work is licensed under a Creative Commons Attribution 4.0 International License. Read Full License 


\section{Ethical Approval}

There is no ethics involved in this article

\section{Consent to participate}

I agree to participate in the study

\section{Consent to Publish}

I consent to publish

\section{Authors Contributions}

This article is written by the first author (Li Wangxu), and the corresponding author (Li Zhenggui) to guide and revise, in the second author (Cheng Jie), the third author (Xu Lianchen), the fourth author (Li Xinrui) and other people's help, jointly completed

\section{Funding}

National Natural Science Foundation, China (Grant No. 52079118), Sichuan Provincial Science and Technology Department Project, Sichuan, China (Grant No. 2020YFH0135), Sichuan Science and Technology Innovation and Entrepreneurship Seedling Project, Sichuan, China (Grant No. 2020043), and The Innovation Fund of Postgraduate (Xihua University), Sichuan, China 
(YCJJ2020045), which are gratefully acknowledged

\section{Competing Interests}

No conflict of interest

\section{Availability of data and materials}

The data and materials in this paper are provided by the

Key Laboratory of Ministry of Education of Xihua University. I guarantee that the test data is true and reliable 
DOI:

\title{
Effect of turbulence intensity of fluid medium interface layer on
}

\section{magnetic liquid seal in liquid environment}

\author{
Wangxu Li ${ }^{a}$, Zhenggui $\mathrm{Li}^{\mathrm{a}}$,b,, Cheng $\mathrm{Jie}^{\mathrm{a}}$, Lianchen $\mathrm{Xu}^{\mathrm{a}}$, Xinrui $\mathrm{Li}^{\mathrm{a}}$ \\ (a. Key Laboratory of Fluid and Power Machinery, Ministry of Education (Xihua University), Chengdu 610039, China; \\ b. Zigong Zhaoqiang Sealing Products Industrial Co., Ltd., Zigong 643000, China)
}

\begin{abstract}
In a liquid environment, the instability of the interface layer of the rotating fluid medium is one of the main causes for the failure of magnetic liquid seals. The turbulence intensity of the interfacial layer between the magnetic and the sealing medium fluids in magnetic liquid seals directly affects the layer stability. Reducing the maximum turbulence intensity is an effective way to improve the stability of the magnetic fluid rotating seal. In this study, we simulated magnetic fluid sealing devices with different structures in liquid environments using FLUENT software. The simulation results are verified through experimental analyses and the turbulence intensity at the sealing interface is analyzed. We simulated the magnetic circuit using Maxwell software, and compared the difference between the optimized and traditional structures. The results show that the maximum turbulence intensity of the liquid interface layer increases with the increasing shaft speed. At the same speed, the turbulence intensity is maximized at the shaft interface before gradually decreasing in a multistage linear pattern along the radial direction. The turbulence intensity at the interface of the spindle is relatively large, which seriously affects the stability of the interface. Based on these results, the optimized structure (OS) of the magnetic liquid seal in the liquid environment is designed. The maximum turbulence intensity of the liquid interface layer in the OS is more than $20 \%$ lower than that in the traditional structure (TS), and it is independent of the rotation speed. The optimized and the traditional structures have the same magnetic induction intensity distribution at the sealing clearance. The maximum magnetic induction intensity of the OS is $6.25 \%$ higher than that of the traditional one. These results provide a reference for designing magnetic liquid sealing devices.
\end{abstract}

Keywords: Liquid environment, magnetic liquid seal, numerical simulation, interface layer, turbulent intensity

\section{Introduction}

In contrast to traditional mechanical seals, magnetic liquid seals have the advantages of zero leakage $e^{[1-2]}$, easy maintenance ${ }^{[3]}$, no pollution, and high reliability ${ }^{[4-}$

\footnotetext{
*Corresponding author. E-mail: Izhgui@mail.xhu.edu.cn
}

The authors would like to thank the National Natural Science Foundation, China (Grant No. 52079118), Sichuan Provincial Science and Technology Department Project, Sichuan, China (Grant No. 2020YFH0135), and Sichuan Science and Technology Innovation and Entrepreneurship Seedling Project, Sichuan, China (Grant No. 2020043) for their financial support.
5]; all of these have attracted the attention of many researchers. The sealing of magnetic liquids against gases has advanced significantly ${ }^{[6]}$, but many problems related to sealing liquids remain worth studying [7]. Kamiyama proposed problems of incompatibility between the magnetic and sealed liquids, as well as issues of liquid-liquid stability when the magnetic liquid is used to seal liquids ${ }^{[8]}$. Van der Wal et al. reported that when sealing and magnetic liquid interfaces are unstable, the liquids emulsify, leading to seal failure ${ }^{[7]}$. Szczech and Horak noted that speed differences between the two liquids caused Kelvin- 
Helmholtz $(\mathrm{KH})$ instability at the two-phase interface, which also led to seal failure ${ }^{[9]}$. Qian proposed an approximate treatment method for liquid interface stability analysis and reported that interface instability is the main cause of seal failure ${ }^{[10]}$. Seal failure, as shown by these reports, is mainly attributed to the instability of the fluid medium interface layer. Therefore, it is necessary to study the factors that cause such instability when the magnetic fluid seals the liquid.

When the interface of two liquids with different densities is subjected to external force, the interface is disturbed and instability occurs. Interface instability is divided into Rayleigh-Taylor (RT) instability caused by gravity fields ${ }^{[11]}$, Richmyer-Meshkov (RM) instability caused by shock waves [12 - 13], and $\mathrm{KH}$ instability caused by speed differences ${ }^{[14]}$. When the magnetic liquid rotates and seals, the rotation of the main shaft drives the rotation of the liquid in the sealed cavity; the speed difference between the magnetic liquid and the liquid in the sealed cavity causes KH instability. In static sealing, no speed difference forms between the magnetic and the sealing liquids and thus no KH instability occurs; therefore, the sealing effect is better. As the rotation speed increases, the nature of the flow between the magnetic liquid and sealing medium changes from laminar to turbulent and the interfacial layer of the fluid medium becomes unstable, immersed, and permeated. The O-shaped sealing ring formed by the magnetic fluid under the action of the magnetic field is damaged, resulting in seal failure. The sealing ring is first damaged near the main shaft, that is, the place where the maximum turbulence intensity of the fluid medium interface layer occurs. Therefore, reducing the maximum turbulence intensity of the interface layer is an effective way to improve the sealing effect of the magnetic liquid.

In this study, a combination of experiments and numerical simulations is used to investigate the traditional magnetic fluid seal structure. Then, the trend of turbulence intensity at the interface layer of the fluid medium as a function of the rotation speed is obtained, the traditional seal structure (TS) is optimized, and the optimized structure (OS) is acquired. The turbulence intensities at the fluid medium interface layers of the TS and OS at different speeds are compared and the simulation results are verified experimentally. Lastly, magnetic field simulation is performed on the TS and OS to verify whether the magnetic circuit of the OS is reasonable.

\section{Theoretical analysis}

Firstly, the internal flow of the sealed liquid is approximated as a three-dimensional incompressible flow. The flow field should satisfy the mass and momentum conservation equations ${ }^{[15]}$.

Mass conservation can be theoretically described as the equality of the mass of the fluid flowing out of the control body to that reduced by the density change in the control body in the same time interval. The formula of the mass conservation equation, or continuity equation, is as follows.

$$
\frac{\partial \rho}{\partial t}+\frac{\partial\left(\rho \mu_{x}\right)}{\partial x}+\frac{\partial\left(\rho \mu_{y}\right)}{\partial y}+\frac{\partial\left(\rho \mu_{z}\right)}{\partial z}=0
$$

Momentum conservation is theoretically described as the equality of the rate of change of the momentum of the control body with respect to time to the sum of various external forces acting on the control body. The formula of the momentum conservation equation is as follows.

$$
\rho \frac{d \vec{\mu}}{d t}=-\nabla P+\nabla \cdot \overrightarrow{\tau_{i j}}+\rho \vec{F}
$$

Turbulence intensity is important for determining the microscopic pulsation characteristics of the flow field and is theoretically represented by the following formula $^{[16]}$ :

$$
I=\frac{u^{\prime}}{\bar{u}}=0.16(R e)^{-\frac{1}{8}}
$$

\section{Numerical simulations}

\subsection{Geometric model}

The turbulence intensity has a significant effect on the 
stability of the interface layer of the fluid medium ${ }^{[10]}$. Considering the complex shape of the actual interface between the magnetic and sealing liquids, and to better extract the relationship of the turbulence intensity of the fluid medium interface layer with the shaft speed, we assume that the magnetic liquid is well sealed, and that the shape of the phase interface between the magnetic and sealing liquids is regular. Then, the two-phase interface can be simplified as a wall surface, and the relationship can be extracted. Figure 1 shows a schematic of the magnetic fluid sealing devices.

In constructing the geometric model, the shaft diameter is taken as $200 \mathrm{~mm}$, the sealing clearance 1 $\mathrm{mm}$, and the pole piece height $40 \mathrm{~mm}$. The OS is slotted on the shaft surface, and the starting end of the slot position is flush with the end of the pole piece. The tested slot depths (SDs) are 1, 2, and 3 times the gap value (1 SD,2 SD, and $3 \mathrm{SD}$ ), and the slot lengths (SLs) are 8,16 , and 32 times the gap value $(8 \mathrm{SL}, 16 \mathrm{SL}$, and $32 \mathrm{SL})$.

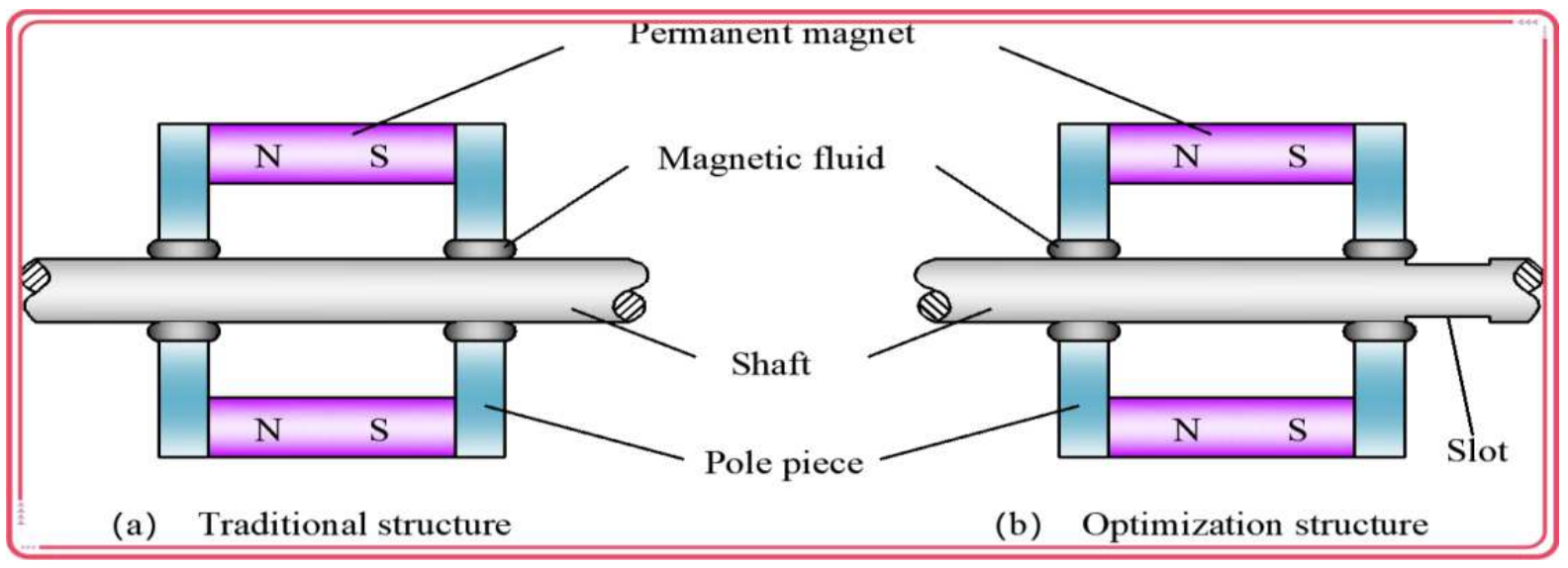

Fig. 1 Schematic of the magnetic fluid sealing devices

\subsection{Mesh model}

The grid model adopts the structural grid drawn using ICEM software and verifies the independence of the grid. Grid independence is obtained from the calculation results; at a speed of $1000 \mathrm{rpm}$, the element number of the TS ( 3 SD, 32 SL ) reaches 229,312, and that of the OS reaches 238,531; the numbers of the monitoring points within the flow field cease changing. It can be considered that these element numbers meet the calculation requirements. All subsequent calculations of the working conditions use grid models with element numbers exceeding these for numerical simulations.

\subsection{Solution control}

The flow field simulation inside the sealed cavity is performed with FLUENT software. The sealing liquid medium is water, and the shaft speeds are set at 100, 300, 500, and $1000 \mathrm{rpm}$. The interface between the magnetic and the sealing liquids is set as the wall surface, and the pressure-velocity coupling adopts the coupled method. The turbulence model is a k-e realizable model. The least-squares cell-based scheme is used for gradient dispersion, and PRESTO! is used for pressure dispersion, while the rest use the second-order upwind scheme.

\section{Results and discussion}

\subsection{Analysis of turbulence intensity at TS interfaces}


When the magnetic liquid rotates in the seal, the liquid in the seal cavity rotates under the action of the shear force resulting from the rotation of the main shaft, forming the flow field shown in Fig. 2. As the rotation speed increases, the flow field in the sealing cavity changes from laminar to turbulent flow. Under turbulent flow, the magnetic liquid is constantly eroded by the sealing medium liquid, and emulsification and mixing easily occur, resulting in sealing failure. To explore the relationship between the turbulence intensity distribution at the interface layer of the fluid medium at the seal gap and the rotational speed, the flow fields at different speeds ranging from 100 to $1000 \mathrm{rpm}$ were simulated. The flow field characteristics at the interface layer of the fluid medium when the magnetic liquid with the TS enters the mobile seal are analyzed quantitatively. As shown in Fig. 3, the turbulence intensity at the

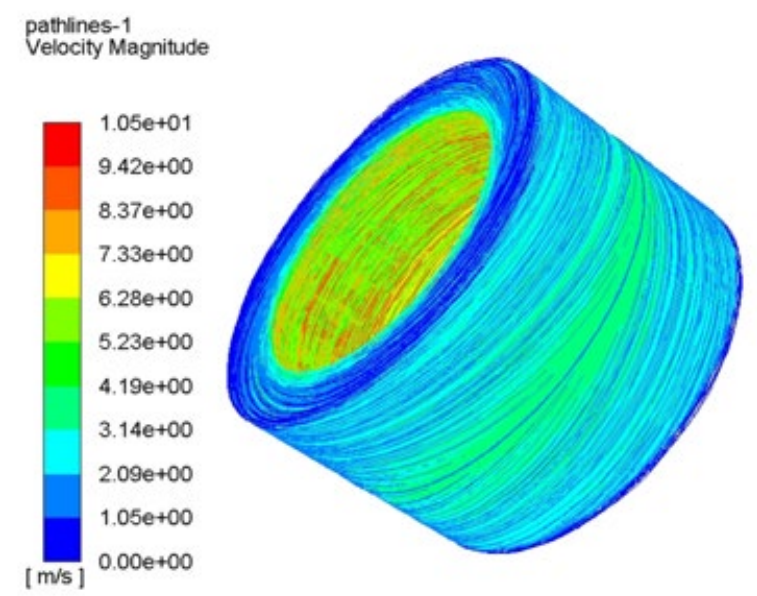

Fig. 2 Flow field diagram in the sealed cavity at 1000

$$
\text { rpm }
$$

interface layer of the fluid medium at the spindle interface in the TS is the maximum at different rotating speeds. From the radial direction of the main shaft to 0.2 times the sealing gap, the turbulence intensity at the interface layer of the fluid medium decreases rapidly, and then tends stabilize. At $0.8-1.0$ times the gap, the turbulence intensity drops again, and reaches the minimum value at the wall. The distribution law of turbulence intensity shows that the multisegment linear pattern of the main shaft decreases along the radial direction. Therefore, the turbulence intensity at the interface of the spindle is the largest and sealing failure is most likely to occur. Reducing the maximum turbulence intensity at the interface layer of the fluid medium at the seal gap is one of the effective methods to improve sealing stability.

To further explore the relationship between the maximum turbulence intensity at the interface layer of the fluid medium in the sealing gap and the rotational speed, we analyzed the maximum turbulence intensity at different rotational speeds ranging from 100 to 1000 rpm. As shown in Fig. 4, the maximum turbulence intensity increases in an approximate linear pattern as the rotating speed increases. Origin software is used to fit the maximum turbulence intensity at different rotating speeds based on the linear curve, and the following relationship can be obtained:

$$
\mathrm{y}=5.089+0.043 \mathrm{x}
$$

Fitting yielded maximum turbulence intensities of $46.0,52.51$, and $69.1 \%$ at 950,1100 , and $1500 \mathrm{rpm}$, respectively. The maximum turbulence intensities calculated using formula (4) are 45.939, 52.389, and $69.589 \%$ at $950,1100,1500 \mathrm{rpm}$, respectively. The fitting results agree well with the numerically calculated ones showing errors less than $1 \%$, thereby proving the correctness of formula (4). 


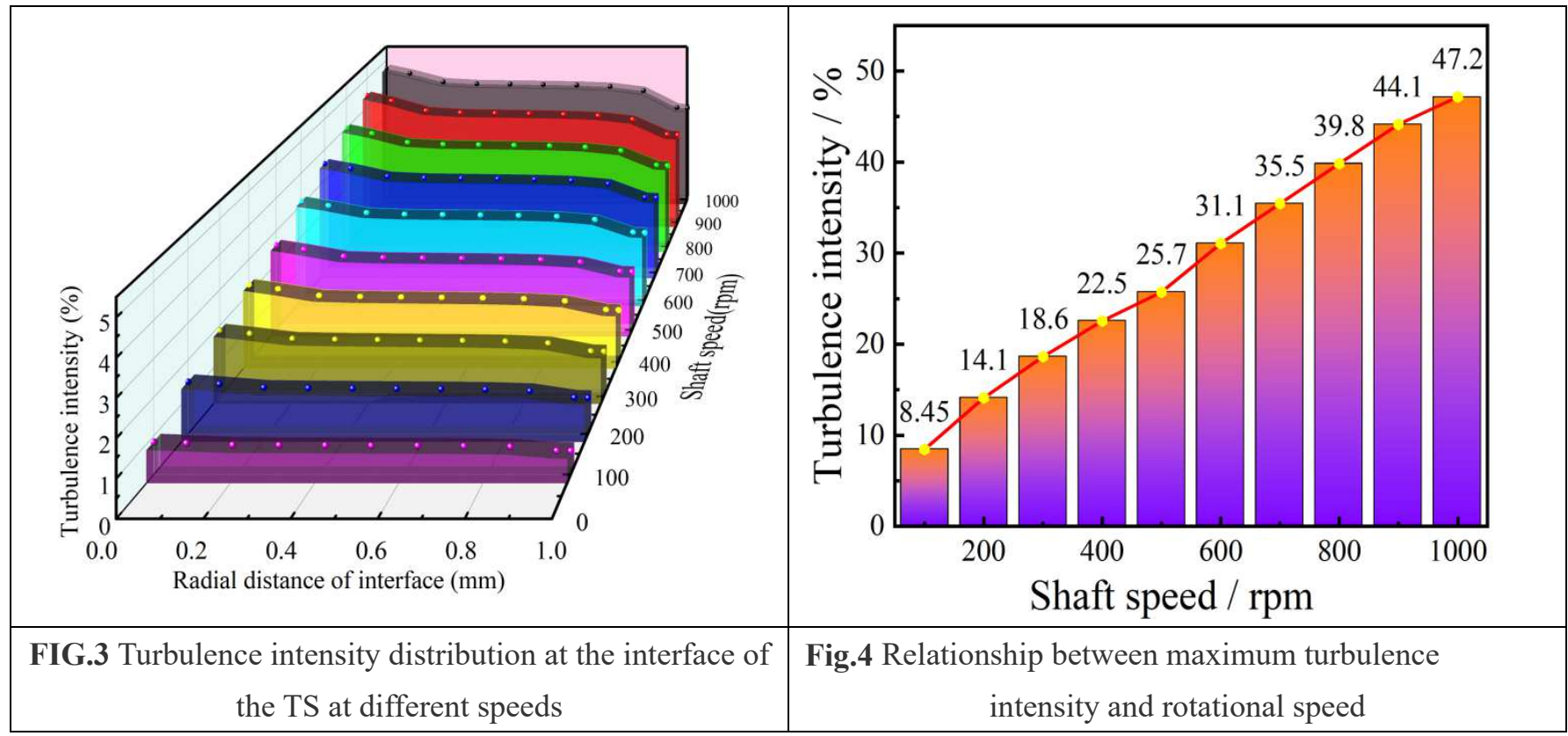

\subsection{Changes of turbulence intensity at the interface with different SDs}

From the above analysis, the maximum turbulence intensity of the TS occurs at the shaft interface. This is mainly because the liquid velocity gradient at this location is the largest, which leads to the largest liquid shear stress, thus resulting in flow turbulence. Reducing the velocity gradient can reduce the turbulence intensity. Placing slots in the shaft of the TS can effectively reduce this velocity gradient and improve the flow regime. To ensure similarity in the experimental variables, the conditions of the sealed liquid material, the sealing clearance, and the shaft speed are held constant; then, the changes in the turbulence intensity of the fluid medium interface layer with different SDs are observed.

As shown in Fig. 5, the grooved structure obviously reduces the maximum turbulence intensity at the interface layer of the fluid medium in the sealing gap. When the slotting length is constant (32 SL), the effect of the groove depth on reducing the maximum turbulence intensity is slightly different at different rotating speeds. When the rotation speed is $100 \mathrm{rpm}$, the effect of $2 \mathrm{SD}$ and $3 \mathrm{SD}$ on reducing the maximum turbulence intensity is higher than that of $1 \mathrm{SD}$; the reduction effect value at $2 \mathrm{SD}$ and $3 \mathrm{SD}$ was approximately 27\% (Effect value = (maximum turbulence intensity of the TS - maximum turbulence intensity of the OS)/maximum turbulence intensity of the TS). When the rotation speeds are 300, 500, and $1000 \mathrm{rpm}$, the reduction effect values at slotting depths 1, 2, and $3 \mathrm{SD}$ are at the same level. At rotating speeds of 300, 500, and $1000 \mathrm{rpm}$, the reduction effect values are approximately $25.8,22.1$, and $22 \%$, respectively.

Comparisons of the results showed that at 100-1000 rpm, $2 \mathrm{SD}$ and $3 \mathrm{SD}$ can reduce the maximum turbulence intensity at the sealing gap by more than $22 \%$. Moreover, after slotting, the rigidity of the spindle will be affected, and it can disturb the flow field in the sealed cavity. The deeper the groove depth, the greater is its influence on the flow field in the sealed cavity; therefore, a groove depth that is two times the sealing gap is the optimum groove depth. 

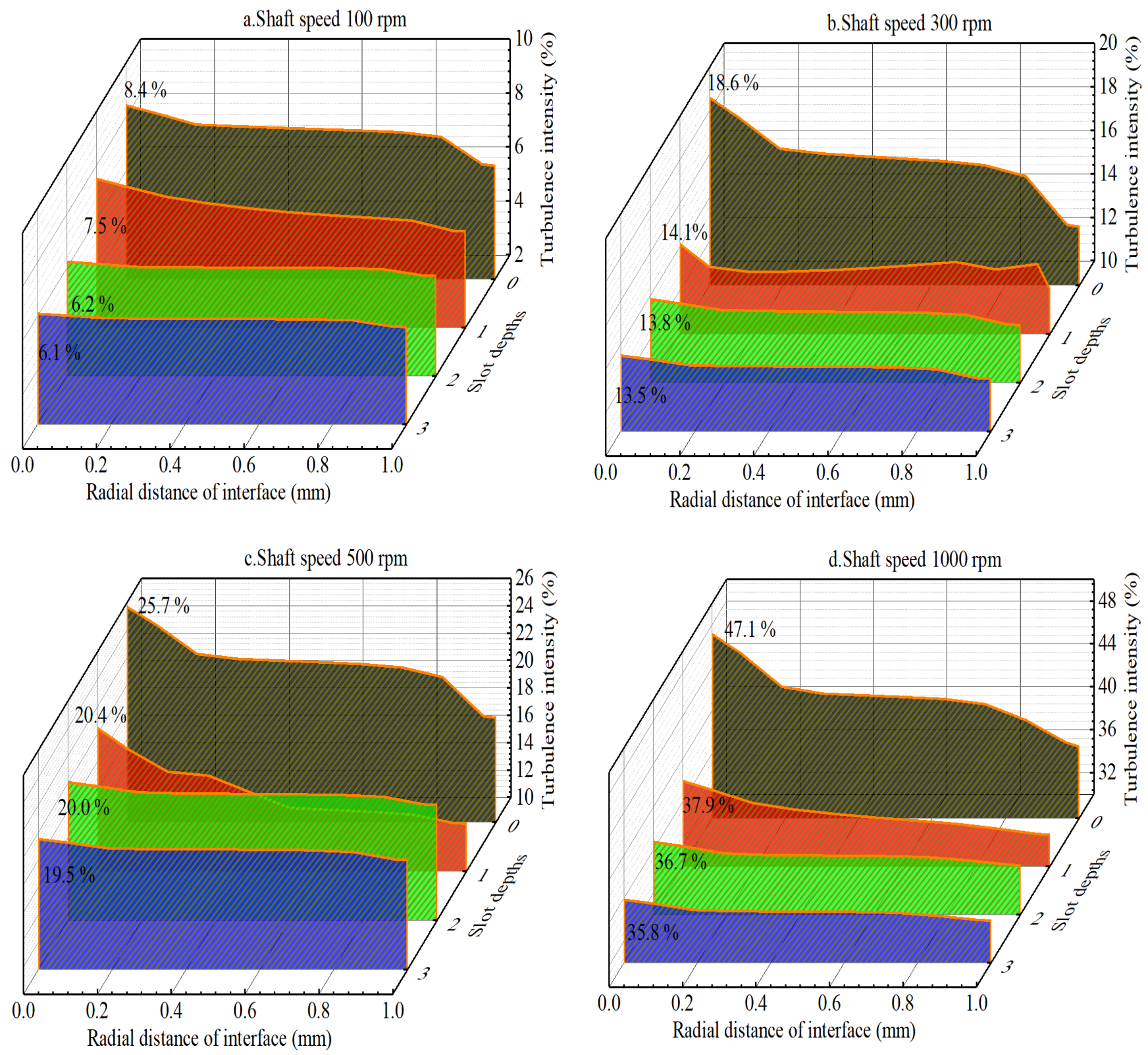

Fig. 5 Turbulence intensity distributions at interfacial layer of the fluid medium under constant speeds and slotting depths

\subsection{Changes in turbulence intensity at the interface with different SLs}

Figure 6 shows that at a certain slotting depth (2 SD), there is little difference in the influences of groove lengths $8 \mathrm{SL}, 16 \mathrm{SL}$, and $32 \mathrm{SL}$ on the maximum turbulence intensity at the interface layer of the fluid medium in the sealing gap at speeds of 100-1000 rpm; the effect values of16 SL and $32 \mathrm{SL}$ are almost the same. When the groove length is $16 \mathrm{SL}$, further increasing it is not conducive to reduce the maximum turbulence intensity at the sealing gap. At $100 \mathrm{rpm}$, the effect value of $8 \mathrm{SL}$ is the same as that of $16 \mathrm{SL}$. At 300, 500, and $1000 \mathrm{rpm}$, the effect values of $8 \mathrm{SL}$ are 2.68, 3.12, and $2.96 \%$ lower than those of $16 \mathrm{SL}$, respectively. To achieve the optimal reduction in the maximum turbulence intensity, the optimal groove length must be 16 times the sealing clearance. 

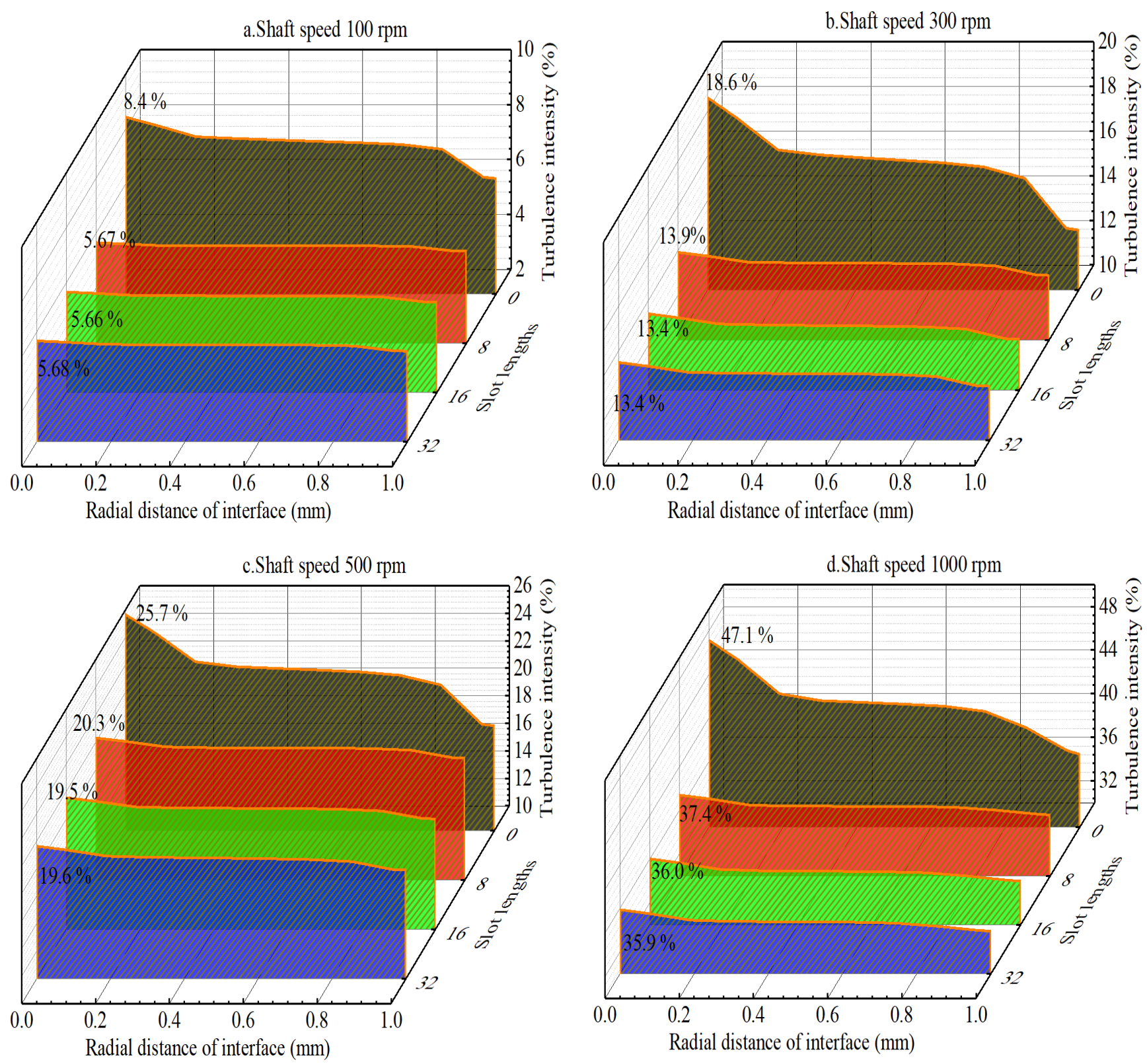

Fig. 6 Turbulence intensity distributions at interfacial layer of the fluid medium under constant speeds and different slotting lengths

4.4 The reduction effect of the optimal slotting structure on the maximum turbulence intensity at

\section{different speeds}

When the optimal slotted structure $(\mathrm{SL}=16 \mathrm{~mm}, \mathrm{SD}$ $=2 \mathrm{~mm}$ ) is adopted, the reduction effect on the maximum turbulence intensity of the fluid medium interface layer at the sealing clearance is shown in Figure 7. At $100 \mathrm{rpm}$, the effect value is the highest at $26.2 \%$. When the rotation speed is above $500 \mathrm{rpm}$, the effect value is stable at approximately $22 \%$. In all the rotational speeds involved, the effect value of the optimal slotting structure to reduce the turbulence intensity at the interface layer of the fluid medium at the seal gap is more than $20 \%$, and the difference is within $5 \%$. The effect of the OS on reducing the turbulence intensity of the interface layer in the fluid medium at the seal gap is not affected by the rotating speed. The optimal groove structure can effectively reduce the maximum turbulence intensity of the interface layer in the fluid medium at the sealing gap. Further, the erosion 
effect of the sealing medium liquid on the O-ring formed by the magnetic fluid in the Magnetic liquid sealing structure is reduced. The sealing gap must be enhanced to improve the sealing stability of the magnetic fluid rotary seal.

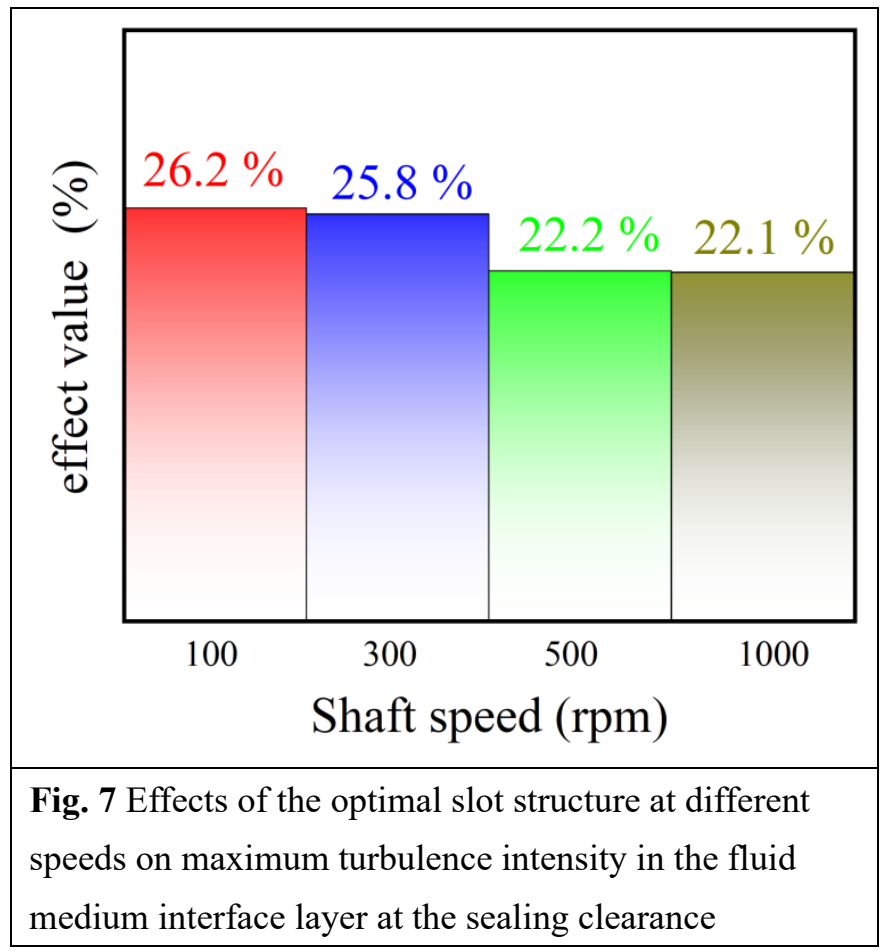

\subsection{Difference between the magnetic fields of OS and} TS

The sealing ability of magnetic liquids mainly depends on the magnetic field strength at the sealing gap and the saturation magnetization of the magnetic liquid

[17]. The magnetic liquid forms an O-shaped sealing ring [18]. The magnetic circuit of the seal can include permanent magnets, pole pieces, the magnetic liquid, and the shaft [19]. A reasonable degree of magnetic circuit design directly affects the magnetic liquid sealing ability.

In comparison to the TS, the OS of the magnetic fluid seal achieves greatly reduced turbulence intensity at the fluid medium interface layer. However, we must verify that the OS does not produce serious magnetic leakage, which would decrease the magnetic induction intensity at the sealing gap.
When the magnetic liquid sealing device achieves static pressure sealing, the pressure difference formula can be simplified as follows ${ }^{[20]}$.

$$
\Delta P=M_{\mathrm{s}} \sum_{i=1}^{N}\left(B_{\max }^{i}-B_{\min }^{i}\right)
$$

It can be seen from equation 5 that the sealing ability of the sealing device is closely related to the magnetic induction intensity of the sealing gap; therefore, it is necessary to detect the magnetic induction intensity at the sealing gap of the OS.

The calculation of the magnetic field is performed using Maxwell software. The pole piece and shaft are made of AISI 1008 steel. The B-H curve of this material is shown in Figure 8. The permanent magnet is $\mathrm{NdFe} 30$. Its residual magnetic flux density $\mathrm{Br}$ is $1.2 \mathrm{~T}$ and the coercivity Hc is $8.38 \times 105 \mathrm{~A} / \mathrm{m}$. Because the magnetic liquid sealing structure is rotating, a two-dimensional (2D) axisymmetric model can be used for calculation. The remaining structural parameters are presented in Table 1. In addition, because the saturation magnetization of the magnetic liquid is almost equal to that of air, the magnetic liquid can be treated as air.

It can be seen from the above that the SL and SD are 16 times and two times the length of the sealing gap, respectively, which are suitable for the magnetic liquid sealing structures at all speeds. The magnetic field at the sealing gap of the OS with these parameters is compared with that of the TS to verify the accuracy of the magnetic circuit design of the OS.

From Figure 9, it is obvious that the magnetic induction intensity distributions at the sealing gap of the OS and TS are generally similar, indicating that the magnetic circuit is designed correctly. After the slots are introduced, the magnetic resistance of the magnetic field in the main shaft part is reduced; thus, the magnetic induction intensity at the sealing gap is enhanced. The maximum magnetic induction intensity at the sealing gap of the OS is increased by $6.25 \%$ compared to that of the TS. 


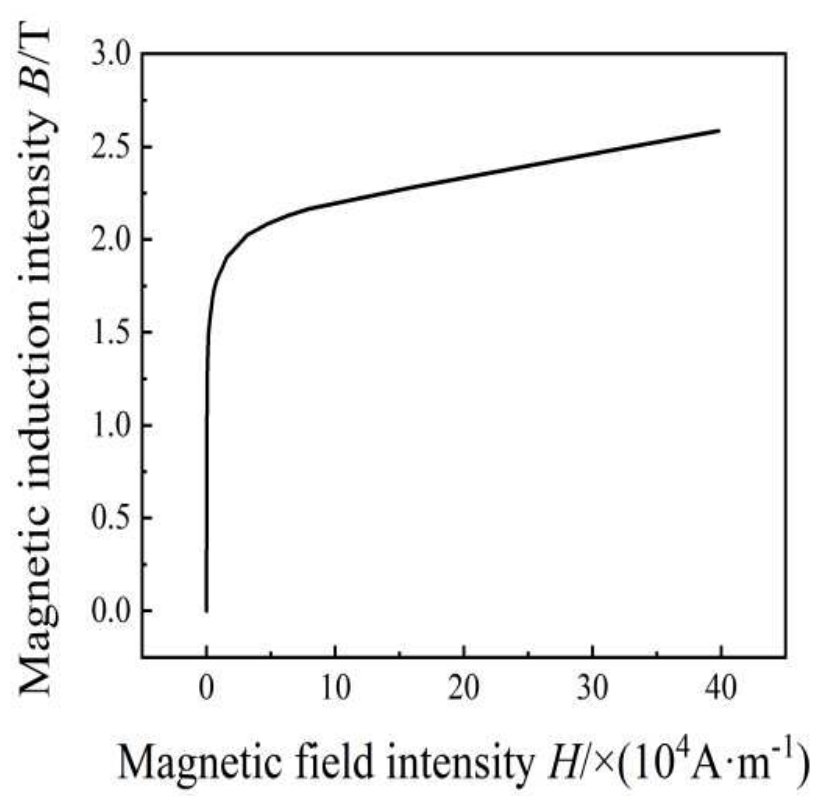

Fig. $8 B-H$ curve of AISI 1008 carbon steel

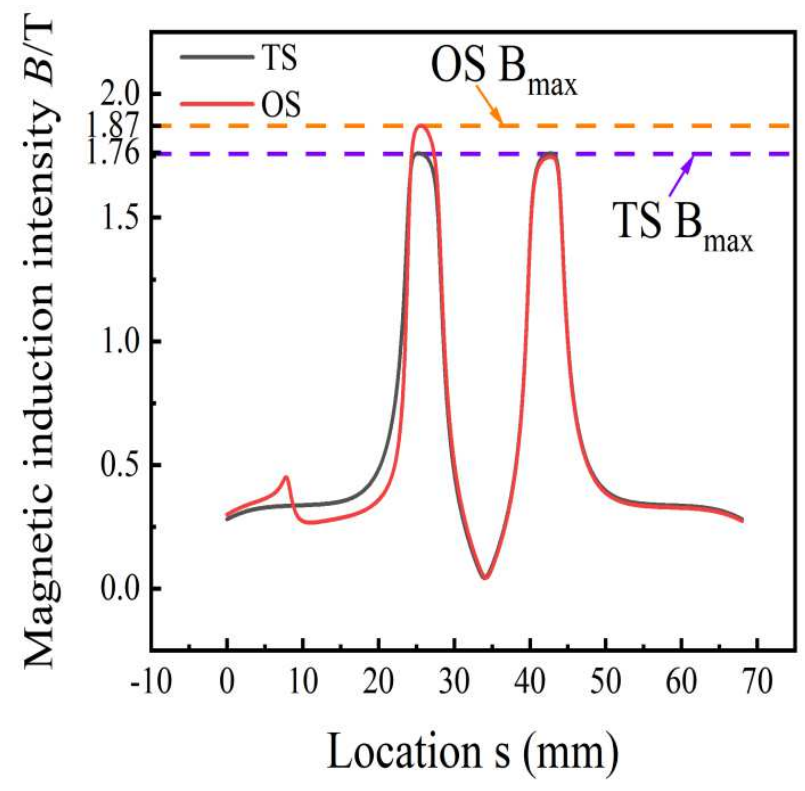

Fig. 9 Contrasts between magnetic induction intensities at the sealing gap

Table 1 Magnetic circuit structure parameters

\begin{tabular}{cc}
\hline Item & Value \\
\hline Diameter of shaft & $200 \mathrm{~mm}$ \\
Inner radius of pole piece & $214 \mathrm{~mm}$ \\
Outer radius of pole piece & $294 \mathrm{~mm}$ \\
Length of pole piece & $28 \mathrm{~mm}$ \\
Height of pole teeth & $6 \mathrm{~mm}$ \\
Width of pole teeth & $4 \mathrm{~mm}$ \\
Angle of pole teeth & $60^{\circ}$ \\
Inner radius of permanent magnet & $214 \mathrm{~mm}$ \\
Outer radius of permanent magnet & $294 \mathrm{~mm}$ \\
Length of permanent magnet & $12 \mathrm{~mm}$ \\
\hline
\end{tabular}

\section{Test validation}

Experimental testing is necessary to validate the simulation results. Therefore, a flow field test experiment was performed in the Key Laboratory of Xihua University. The experimental device adopts the 2D Particle Image Velocimetry (PIV) system purchased by Xihua University from ILA in Germany. The system is suitable for non-contact probing of the $2 \mathrm{D}$ velocity field on a plane of the fluid. To obtain an accurate measurement, the main function parameters are shown in Table 2.

Table 2 Main technical parameters of PIV system

\begin{tabular}{cc}
\hline Main parameters & Technical indices \\
\hline Function & Measure the 2D velocity field distribution on a plane to
\end{tabular}


obtain $\mathrm{U}, \mathrm{V}$ velocity components, vorticity, velocity

gradients, streamlines, etc.

Speed measurement range

Speed measurement accuracy

Measured area

Overall operating frequency

Bits

$$
0-1000 \mathrm{~m} / \mathrm{s}
$$

$\mathrm{U}, \mathrm{V}$ component $\leq 1 \%$ of reading

Flow field area $\geq 900 \mathrm{~mm} \times 1000 \mathrm{~mm}$

$15 \mathrm{~Hz}$

16 bits
The PIV system consists of a dual-cavity dual-pulse laser, a guarding light arm, a sheet-light-source lens group, a high-speed camera, a synchronizer, a tracer particle generator, PIV image analysis software, and a computer. The high-speed camera for PIV is the ILA.PIV.sCMOS and the laser is the LPS PIV200.

The test device comprises the PIV system, a generator, a rotating shaft, and a sealed cavity constructed using transparent acrylic material. Figure 10 is a schematic of the test device. The pulsed laser beam passes through the lens group to form a piece of the light source, and the laser illuminates the measurement area in the flow

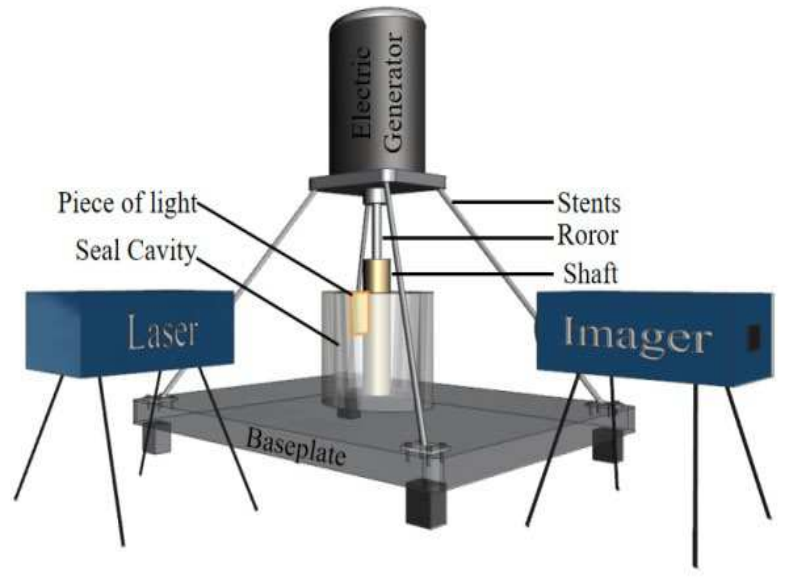

Fig. 10 Schematic of the test device

Because the sealing clearance is narrow, it is difficult to accurately obtain the velocity distribution of the fluid at the sealing clearance; thus, it is also difficult to measure the turbulence intensity at the sealing gap using the test method. In this study, we test the velocity distributions on a horizontal line in the sealed cavity at different spindle speeds and compare these with the field, which is a very thin $(\leq 1 \mathrm{~mm})$ layer of the field. The camera is perpendicular to the direction of the light for imaging. Two consecutive images are obtained at a short interval. The images include the trajectory of the tracer particles in the flow field. The obtained pictures are processed in the computer using PIV special software. The software uses cross-correlation technology for image processing. Calculating the $\mathrm{dx}$ and dy displacements of the tracer particles within a known time yields the distribution of the $2 \mathrm{D}$ velocity in the flow field.

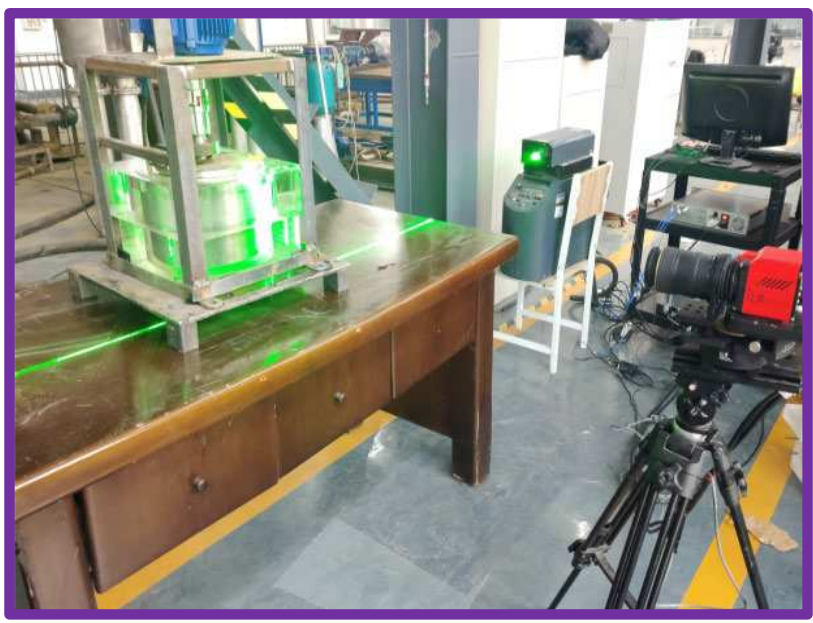

Fig. 11 Field test set-up

numerical simulation results to verify the correctness of the flow field (Figure 11 shows the PIV flow field test experiment in the Key Laboratory of Fluid and Power Machinery, Ministry of Education, Xihua University). After removing invalid test data and smoothing the curve, the experimental data and simulation data exhibited the same general trend but with some 
differences. As shown in Figure 12, the differences are mainly based on the following aspects:

(1) In the test, motor vibration during operation affects the fluid flow in the sealed cavity, thus influencing the test results.

(2) The surface roughness of the main shaft and that of the sealed cavity are not consistent with the parameter values in the simulation software.

(3) The machining problems during shaft design resulted in a slight eccentricity of the shaft.

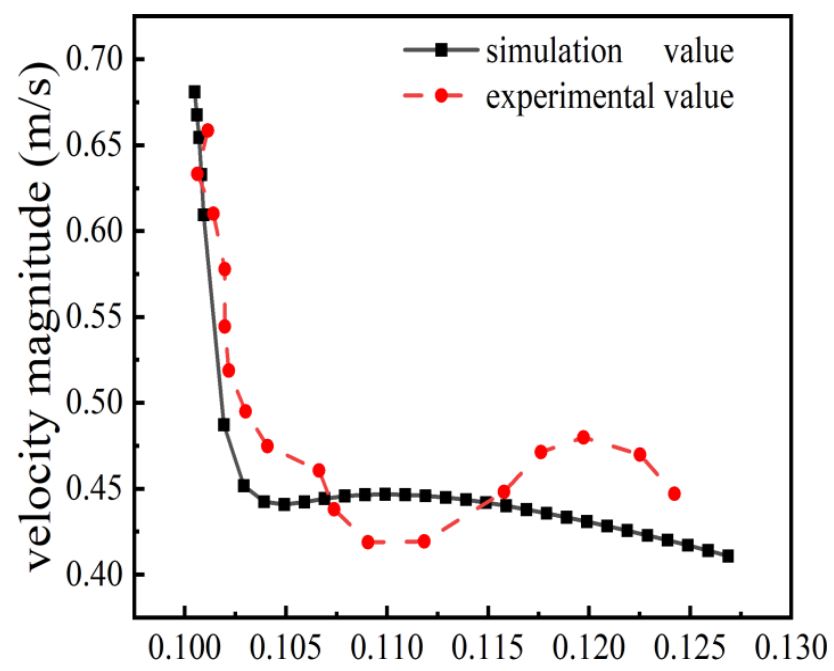

(a) Location's (m)

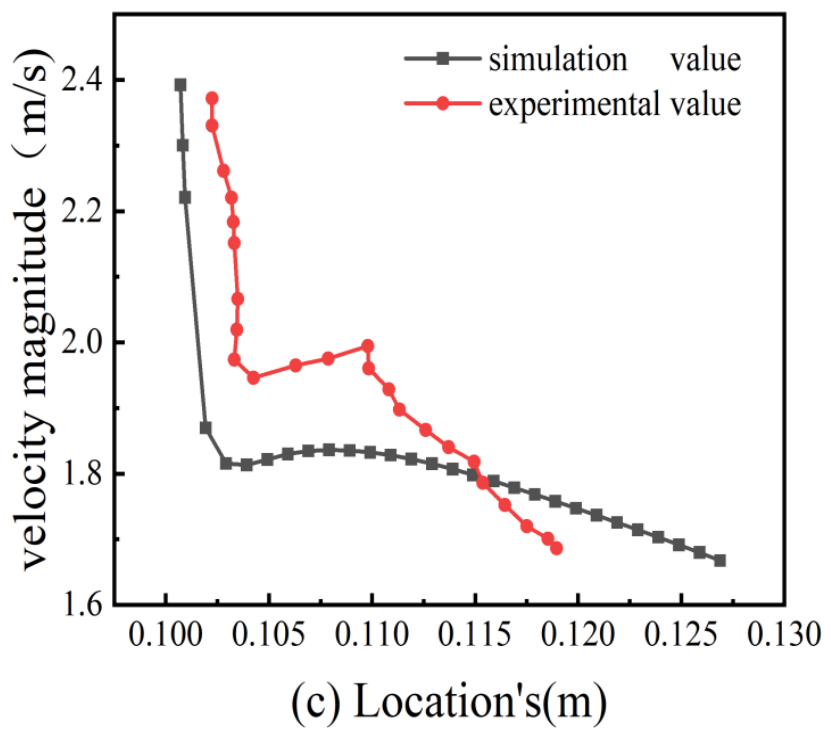

(4) Because the inside of the sealed cavity is a circular wall, refraction will occur when the cavity is irradiated with the laser and the camera records the images.

(5) To simplify the calculations, the software program automatically makes many assumptions during the simulation.

However, the test and simulation values roughly exhibit the same general trend, indicating that the simulation results are credible to a certain extent.

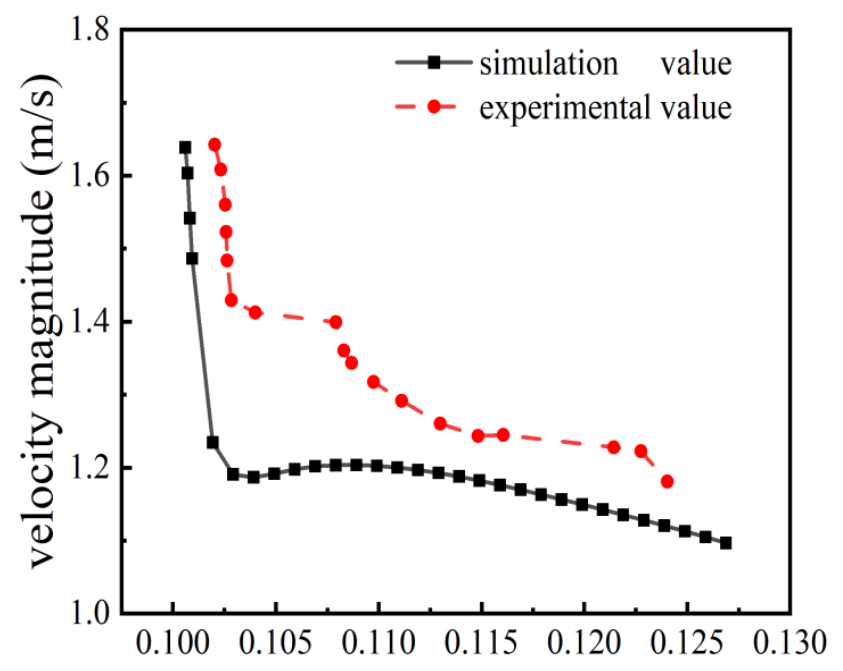

(b) Location's (m)

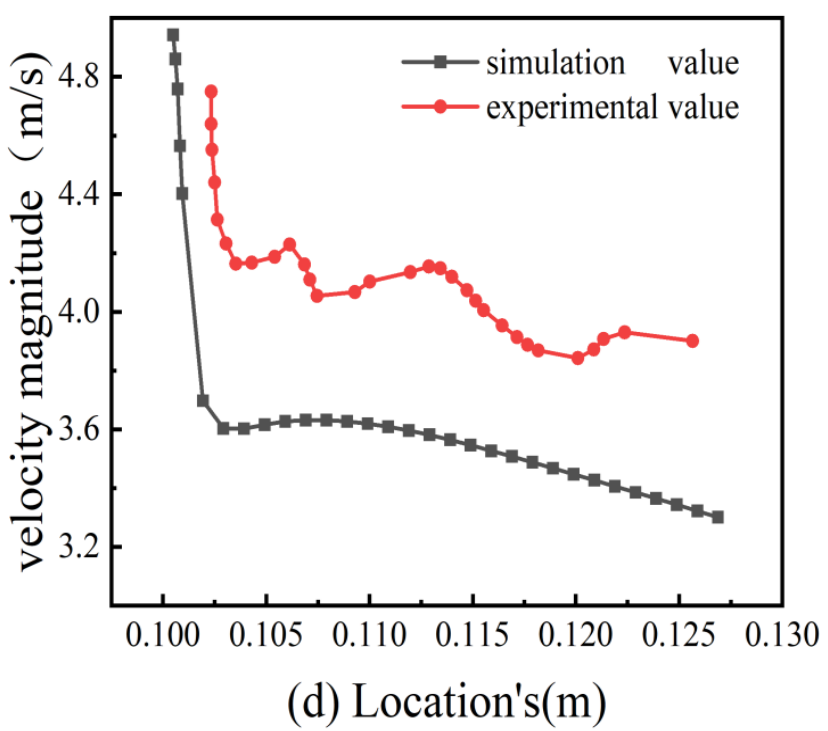

Fig. 12 Comparison of experimental and simulation data ((a) $\omega=100 \mathrm{r} / \mathrm{min}$, (b) $\omega=300 \mathrm{r} / \mathrm{min}$, (c) $\omega=500 \mathrm{r} / \mathrm{min}$, and (d) $\omega=1000 \mathrm{r} / \mathrm{min})$

\section{Conclusions}


The turbulence intensity distribution in the liquid medium at the interface layer of the sealing gap in the magnetic fluid rotary seal shows a multistage linear decreasing trend along the radial direction of the main shaft. The maximum turbulence intensity at the seal clearance has a linear relationship with the rotational speed, expressed as $\mathrm{y}=5.089+0.043 \mathrm{x}$ (applicable within $1500 \mathrm{rpm})$.

Structural grooving has obvious effects on reducing the maximum turbulence intensity at the seal clearance. The calculation results show that the optimal groove size is 16 times the structural groove length and 2 times the structural groove depth.

\section{Nomenclature}

\section{$t$}

$\rho$

$\mu_{\mathrm{x}}, \mu_{\mathrm{y}}, \mu_{\mathrm{z}}$

and $P$

$\overrightarrow{\tau_{\mathrm{ij}}}$

$\vec{F}$

$\bar{\mu}$

$\mu^{\prime}$

$M_{s}$

$\mu_{0}$

$B_{\max }^{i}, B_{\min }^{i}$

$\boldsymbol{R e}$

\section{References}

1.Yang X, Sun P, Hao F (2020) Magnetic field finite element analysis of diverging stepped ferrofluid seal with a large gap and two magnetic sources. Int J Appl Electromagn Mech 63:31-44. 10.3233/JAE-190127

2.Merklein M, Rösel S (2010) Characterization of a magnetorheological fluid with respect to its suitability
At rotating speeds of 100-1000 rpm, the optimal groove structure reduces the maximum turbulence intensity at the seal clearance by approximately $20 \%$.

Because the Magnetic liquid sealing device clearance is small and opaque, the flow state of the Magnetic liquid sealing device itself in the seal clearance is not fully considered. In our future work, we intend to study the magnetic fluid flow field during the operation of the rotary seal by mixing the magnetic fluid with a fluorescent agent.

These research results provide a theoretical reference for the design and operation of magnetic liquid seals in liquid environments.

\section{Per unit time}

Fluid density

Velocity components of the fluid along the $\mathrm{x}, \mathrm{y}$, and $\mathrm{z}$ axes

Fluid pressure

Viscous shear stress on fluid

Volume force on the fluid

Average velocity of fluid

Root mean square of the fluid pulsating velocity

Saturation magnetization of magnetic fluid

Vacuum permeability

Maximum magnetic induction intensity under the level-I pole tooth in the sealing device

Reynolds number

for hydroforming. Int $\mathrm{J}$ Mater Form 3:283-286. https://doi.org/10.1007/s12289-010-0762-8

3.Biao X, Yiping L, Hongjuan R (2014) Review on magnetorheological fluid and its application. Am J Nanosci Nanotechnol 2:70-74.

4.Moskowitz R (1975) Dynamic sealing with magnetic fluids. ASLE Trans 18:135-143. https://doi.org/10.1080/05698197508982756 
5.Zhou H, Zhao W, Zhang H, Wang Y, Wu X, Sun Z (2020) Magnetorheological seal: A review. Int J Appl Electromagn Mech 62:763-786. 10.3233/JAE-190082 6.Liu T, Cheng Y, Yang Z (2005) Design optimization of seal structure for sealing liquid by magnetic fluids. J Magn Mater 289:411-414. https://doi.org/10.1016/j.jmmm.2004.11.116

7.van der Wal K, van Ostayen RAJ, Lampaert SGE (2020) Ferrofluid rotary seal with replenishment system for sealing liquids. Tribol Int 150. https://doi.org/10.1016/j.triboint.2020.106372

8.He X, Miao Y, Li W, Li D (2019) Latest development in sealing of liquid medium with magnetic fluid. Chin $\mathrm{J}$ Vac Sci Technol 39:361-366.

9.Szczech M, Horak W (2015) Tightness testing of rotary ferromagnetic fluid seal working in water environment. Ind Lubr Tribol 67:455-459. https://doi.org/10.1108/ILT-02-2015-0014

10.Qian J (2009) Study on stability of dynamic interface in magnetic fluid seal liquid. Dissertation, China University of Mining and Technology

11.Raj N, Karthick S (2021) Characterization of Rayleigh-Taylor instability at the fluid-fluid interface. Trends Mech Biomed Des:401-415. ttps://doi.org/10.1007/978-981-15-4488-0-35

12.Zhang HH, Zheng C, Aubry N, Wu WT, Chen ZH. (2020) Numerical analysis of Richtmyer-Meshkov instability of circular density interface in presence of transverse magnetic field. Phys Fluids 32. https://doi.org/10.1063/5.0021980

13.Cao J, Wu Z, Ren H, Li D (2008) Effects of shear flow and transverse magnetic field on Richtmyer-Meshkov instability. Phys Plasmas 15. https://doi.org/10.1063/1.2842367

14.Völkel A, Kögel A, Richter R (2020) Measuring the Kelvin-Helmholtz instability, stabilized by a tangential magnetic field. J Magn Mater 505. https://doi.org/10.1016/j.jmmm.2020.166693

15.Foroutan H, Yavuzkurt S (2013) Partially averaged Navier-Stokes modeling of turbulent swirling flow. APS $1 \mathrm{~A}, \mathrm{p} 039$.

16.Fang J, Zheltovodov AA, Yao Y, Moulinec C, Emerson DR (2020) On the turbulence amplification in shockwave/turbulent boundary layer interaction. J Fluid Mech 897. https://doi.org/10.1017/jfm.2020.350

17.Meng Z, Jibin Z, Jianhui H (2006) An analysis on the magnetic fluid seal capacity. J Magn Magn Mater 303:e428-e431.

https://doi.org/10.1016/j.jmmm.2006.01.060

18.Szczęch M (2020) Magnetic fluid seal critical pressure calculation based on numerical simulations. Simulation 96:403-413.

19.Qian JG, Yang ZY (2008) Characteristics of a magnetic fluid seal and its motion in an axial variable seal gap. J China Univ Min Technol 18:634-636. https://doi.org/10.1016/S1006-1266(08)60308-9

20. Yang X, Wang G, Zhang R (2020) Magnetic circuit design and magnetic field finite element analysis of converging stepped magnetofluid seal with small clearance. Adv Mater Sci Eng 2020:1-10. https://doi.org/10.1155/2020/4201891 
Figures

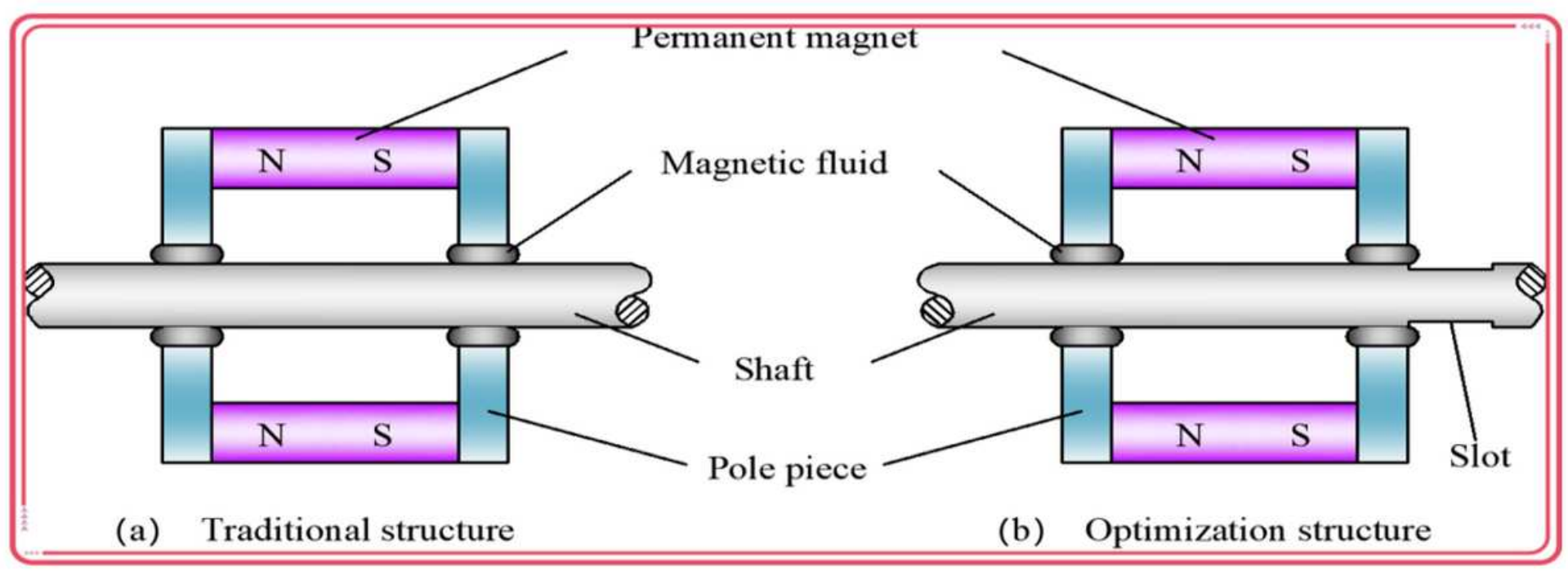

Figure 1

Schematic of the magnetic fluid sealing devices

pathlines-1

Velocity Magnitude
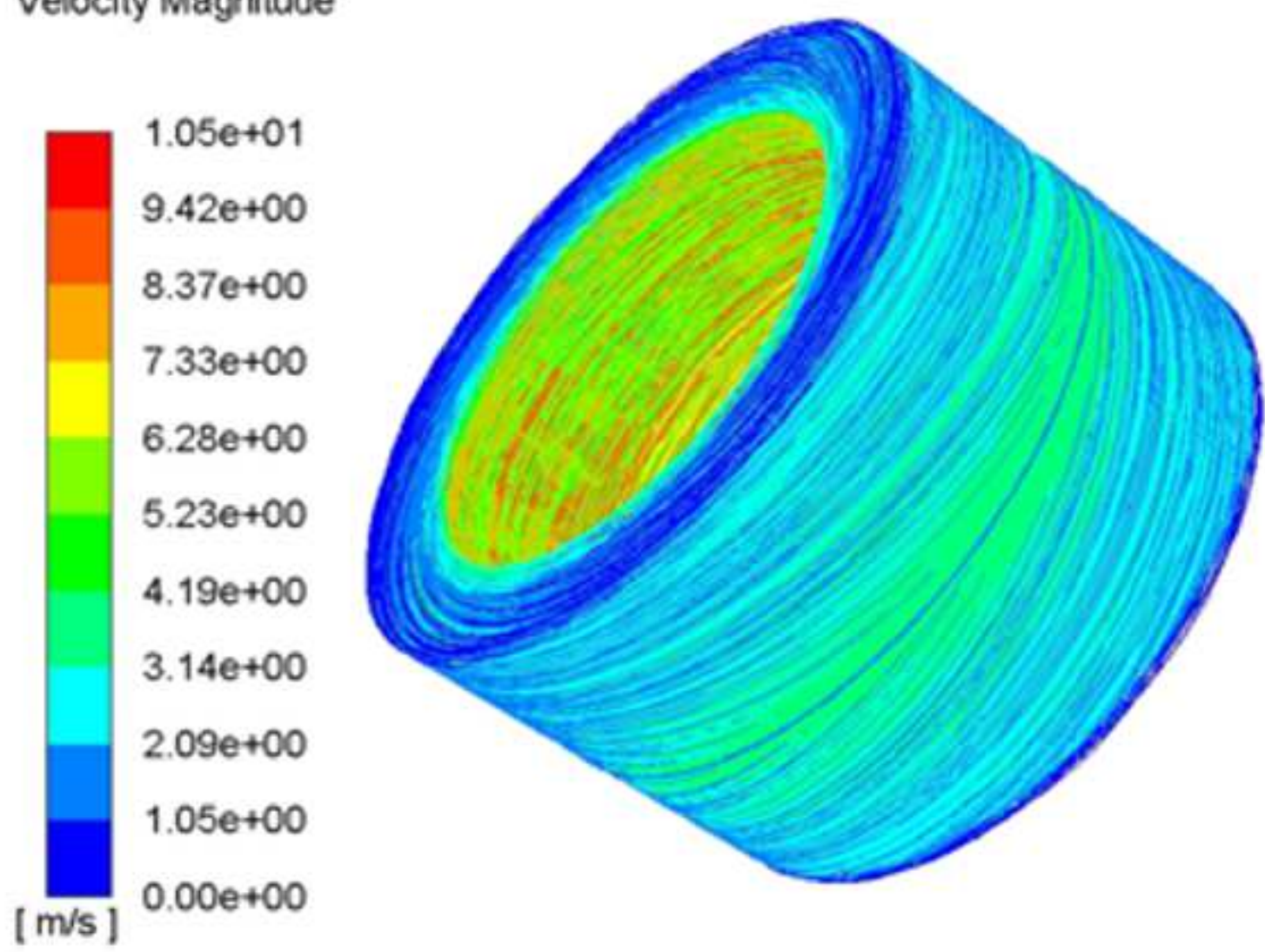
Figure 2

Flow field diagram in the sealed cavity at $1000 \mathrm{rpm}$

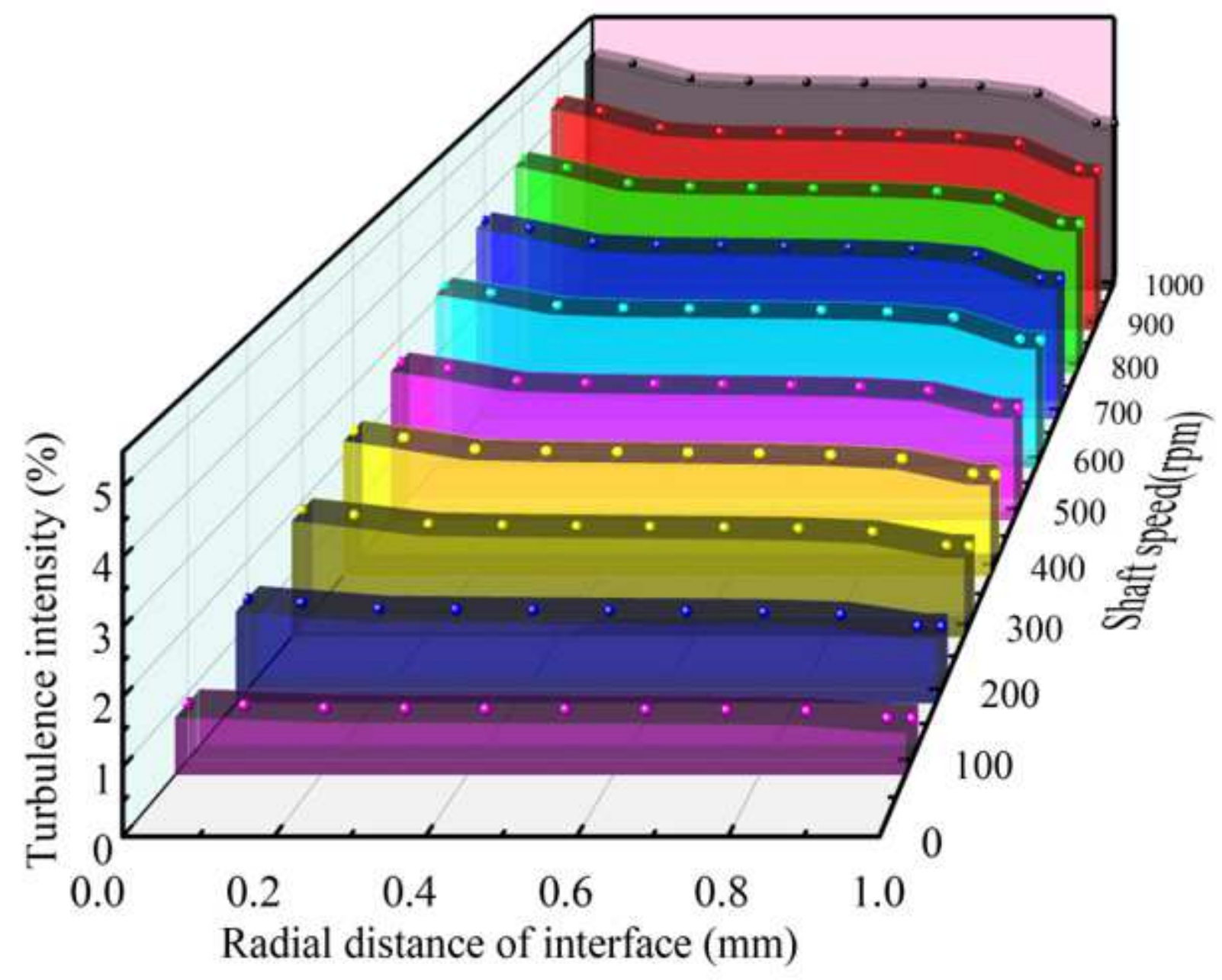

Figure 3

Turbulence intensity distribution at the interface of the TS at different speeds 


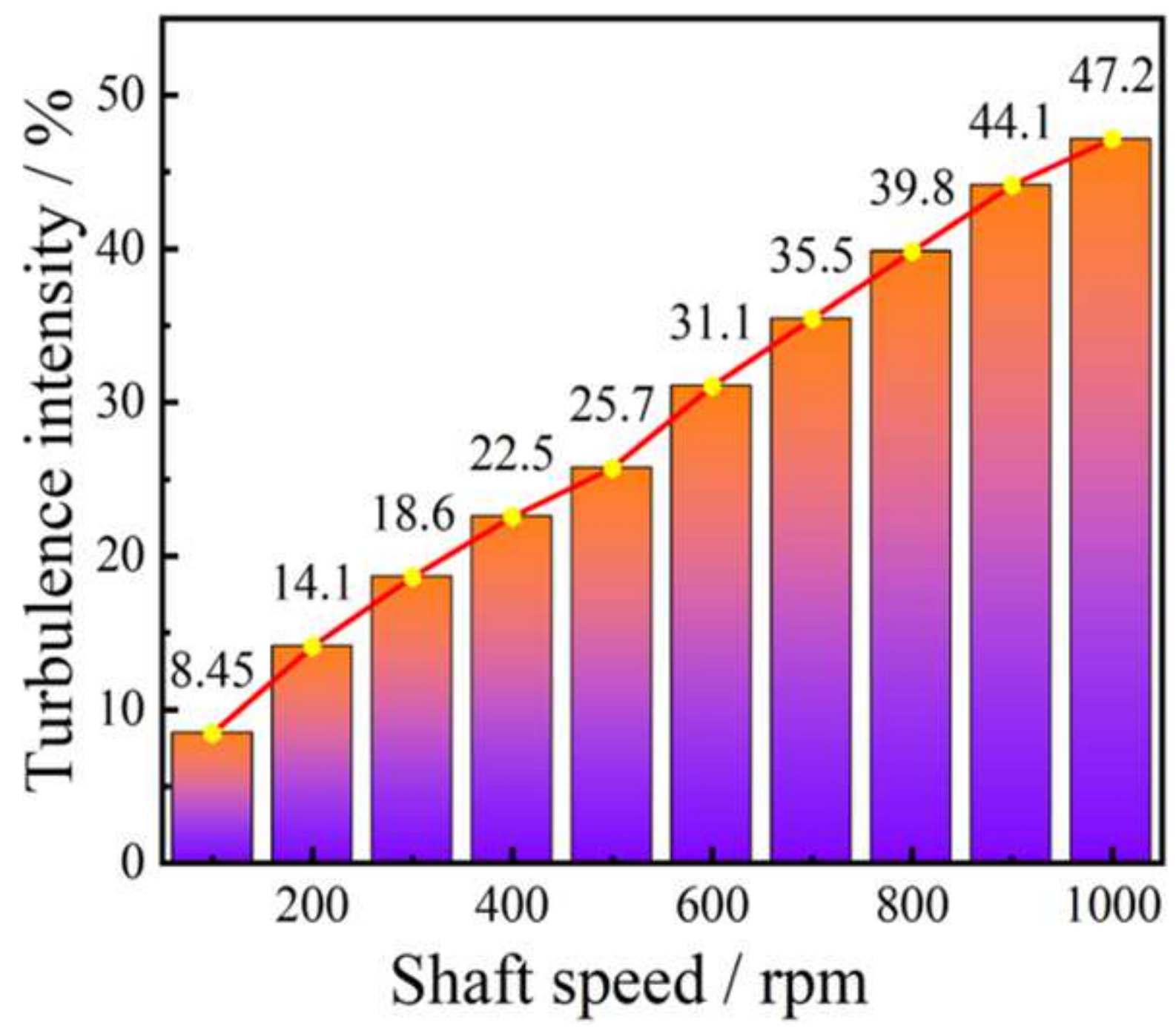

Figure 4

Relationship between maximum turbulence intensity and rotational speed 

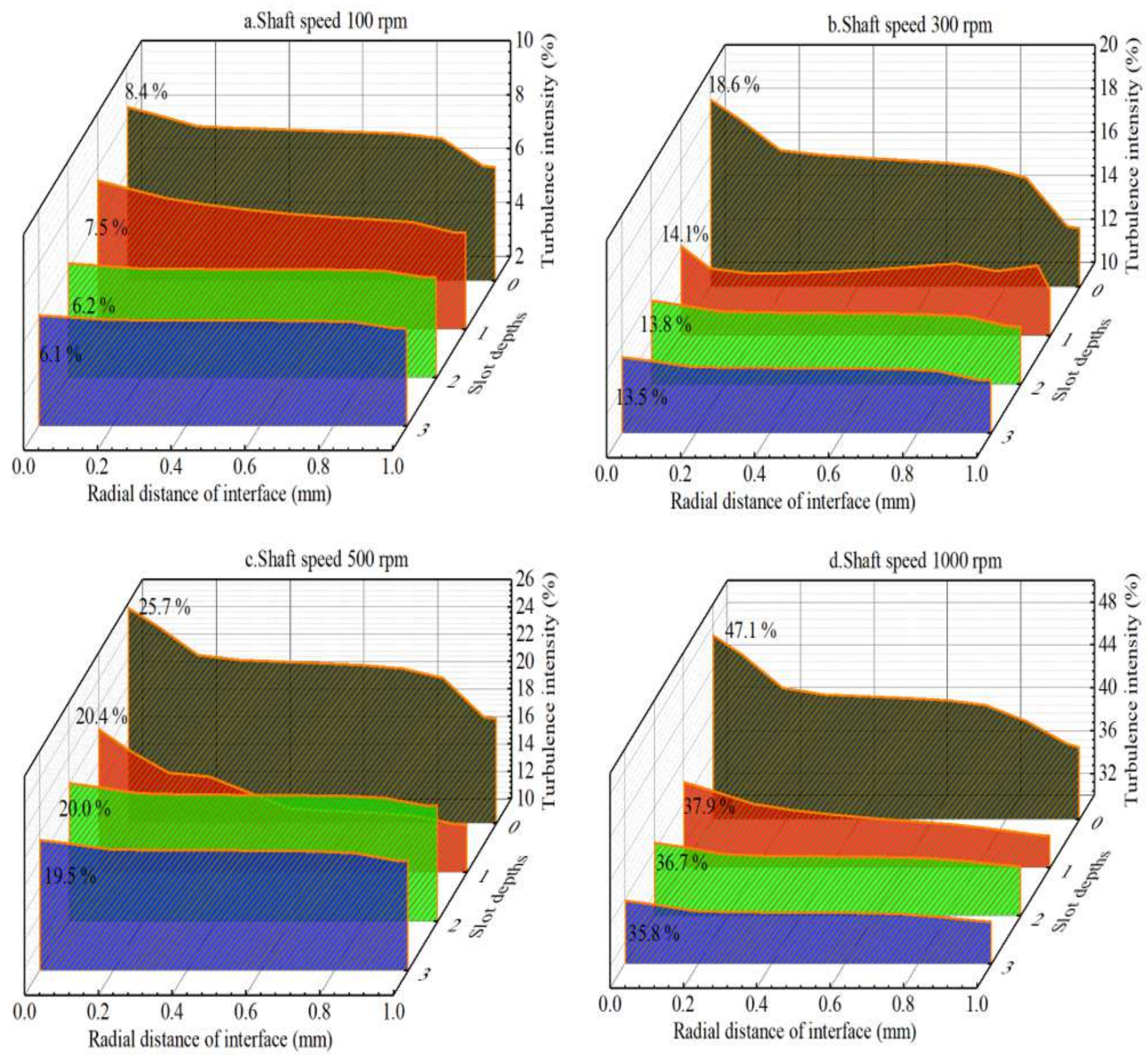

Figure 5

Turbulence intensity distributions at interfacial layer of the fluid medium under constant speeds and slotting depths 

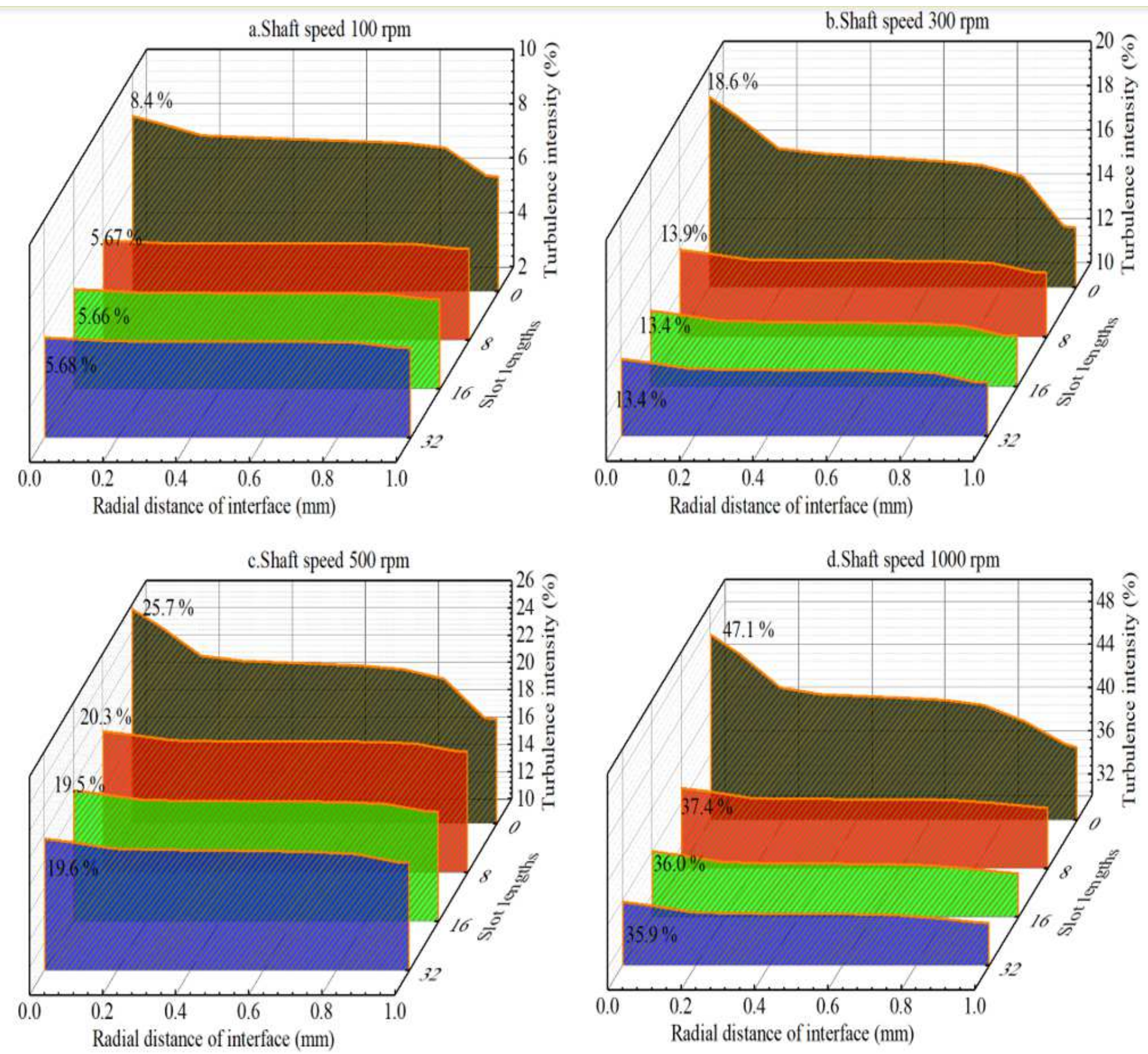

Figure 6

Turbulence intensity distributions at interfacial layer of the fluid medium under constant speeds and different slotting lengths 


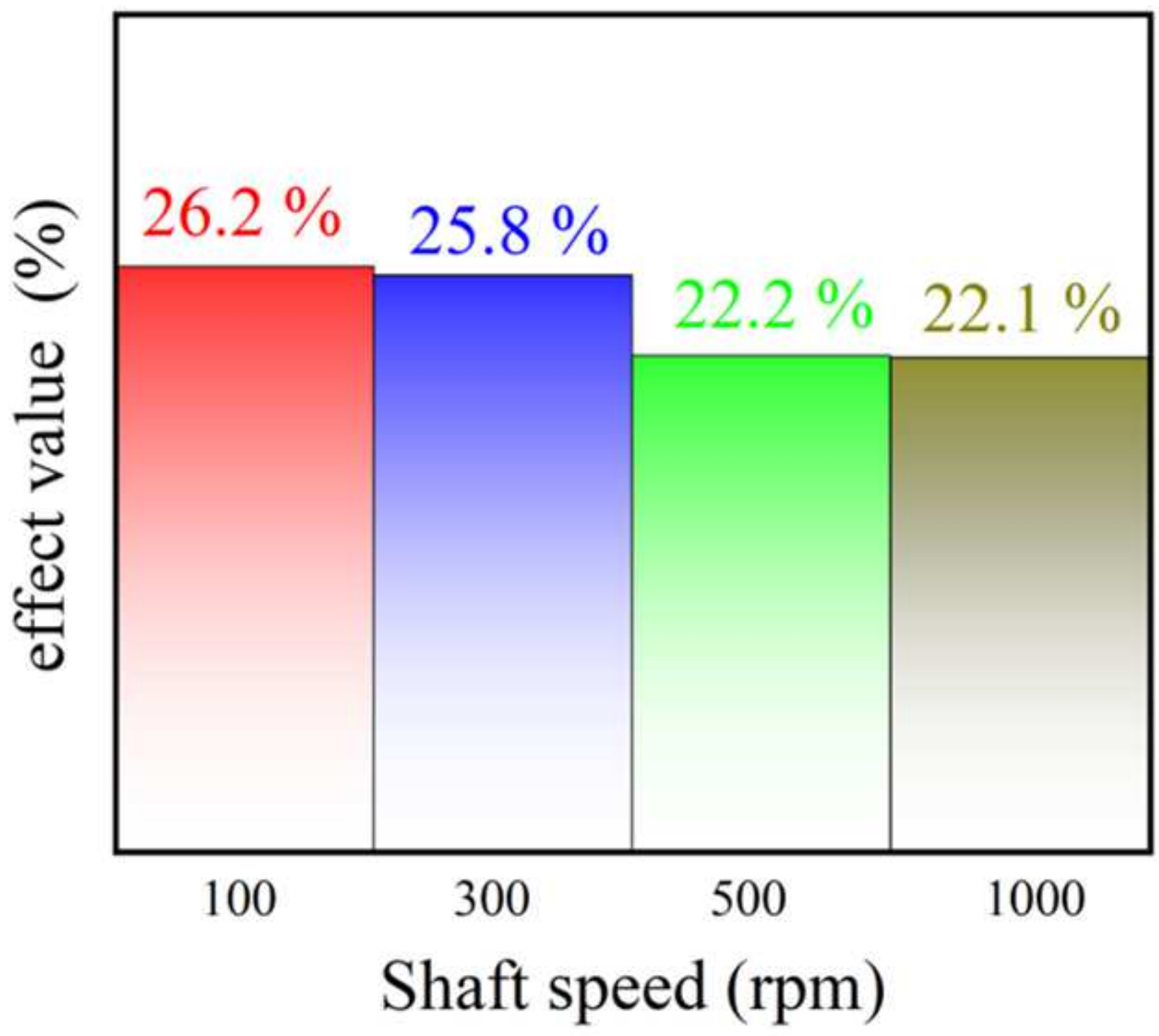

Figure 7

Effects of the optimal slot structure at different speeds on maximum turbulence intensity in the fluid medium interface layer at the sealing clearance 


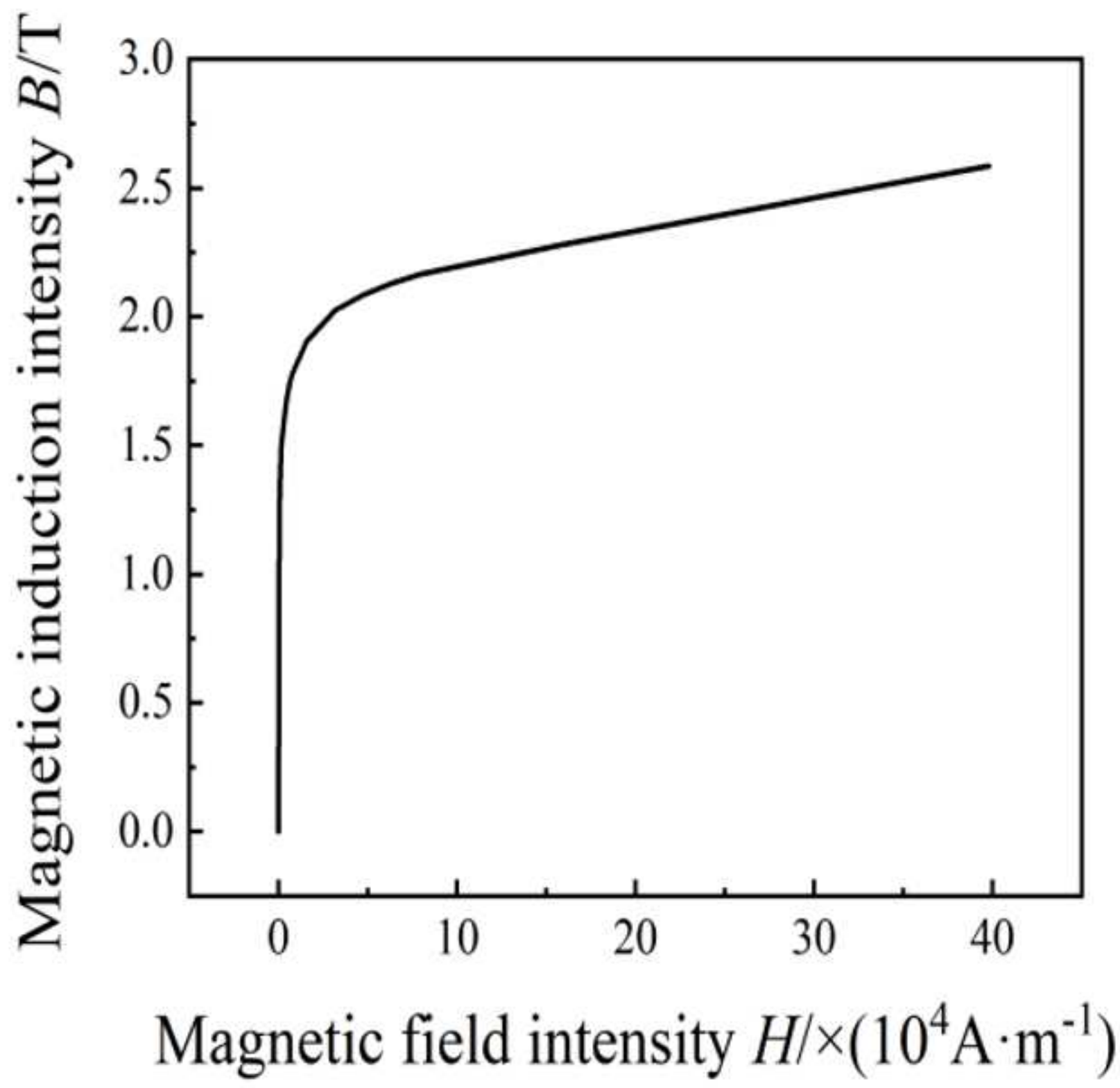

Figure 8

B-H curve of AISI 1008 carbon steel 


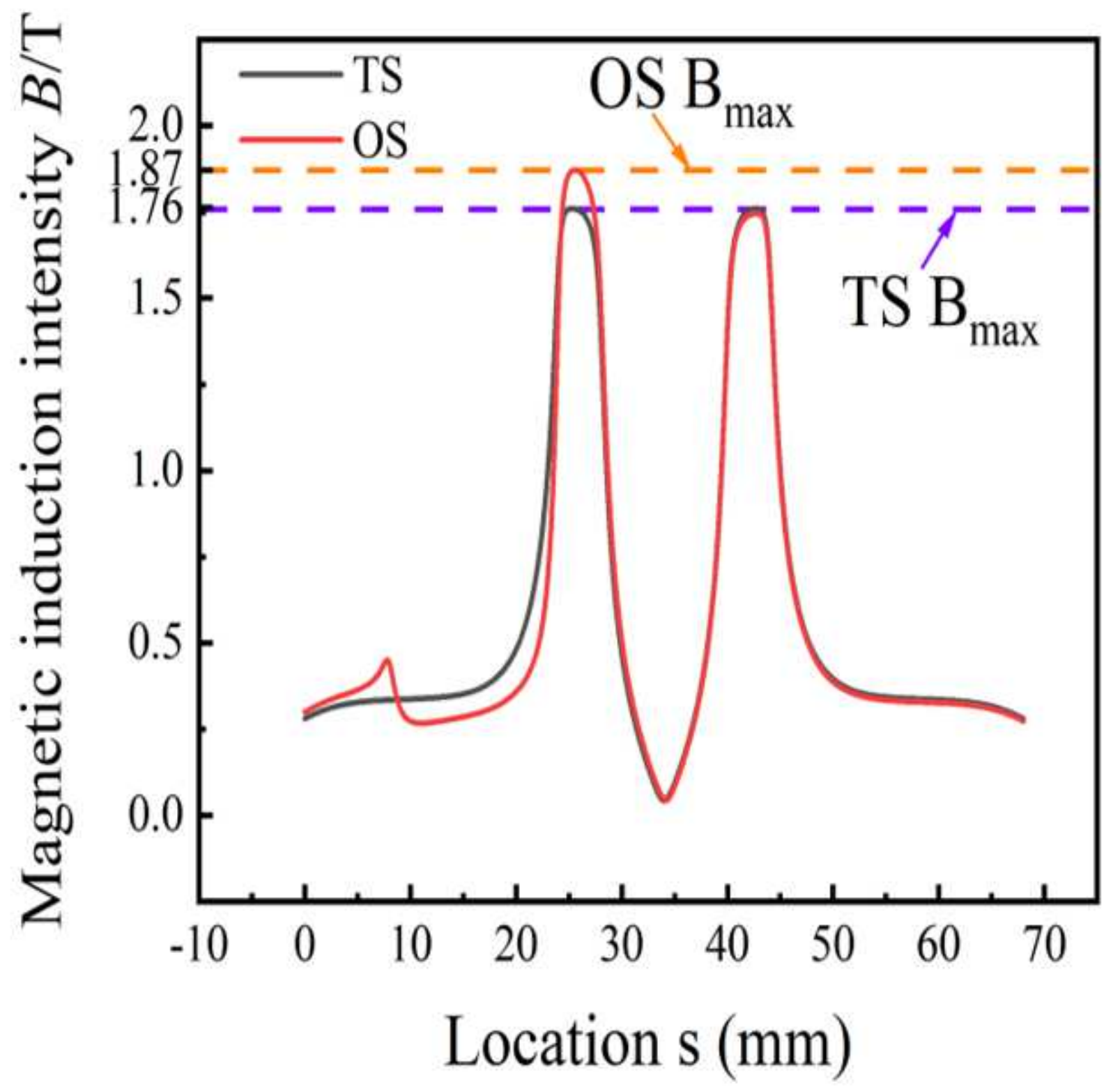

Figure 9

Contrasts between magnetic induction intensities at the sealing gap 


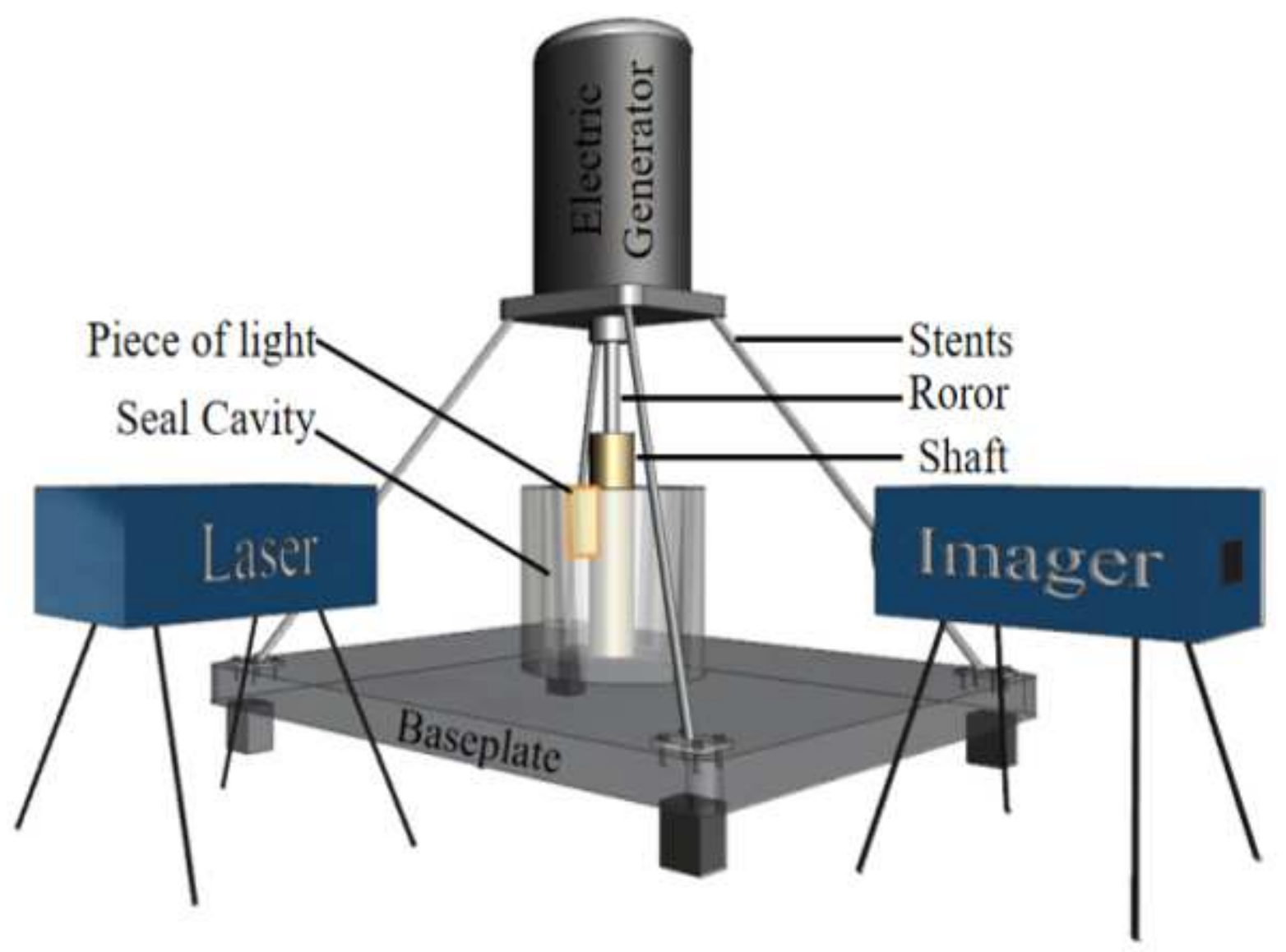

Figure 10

Schematic of the test device 


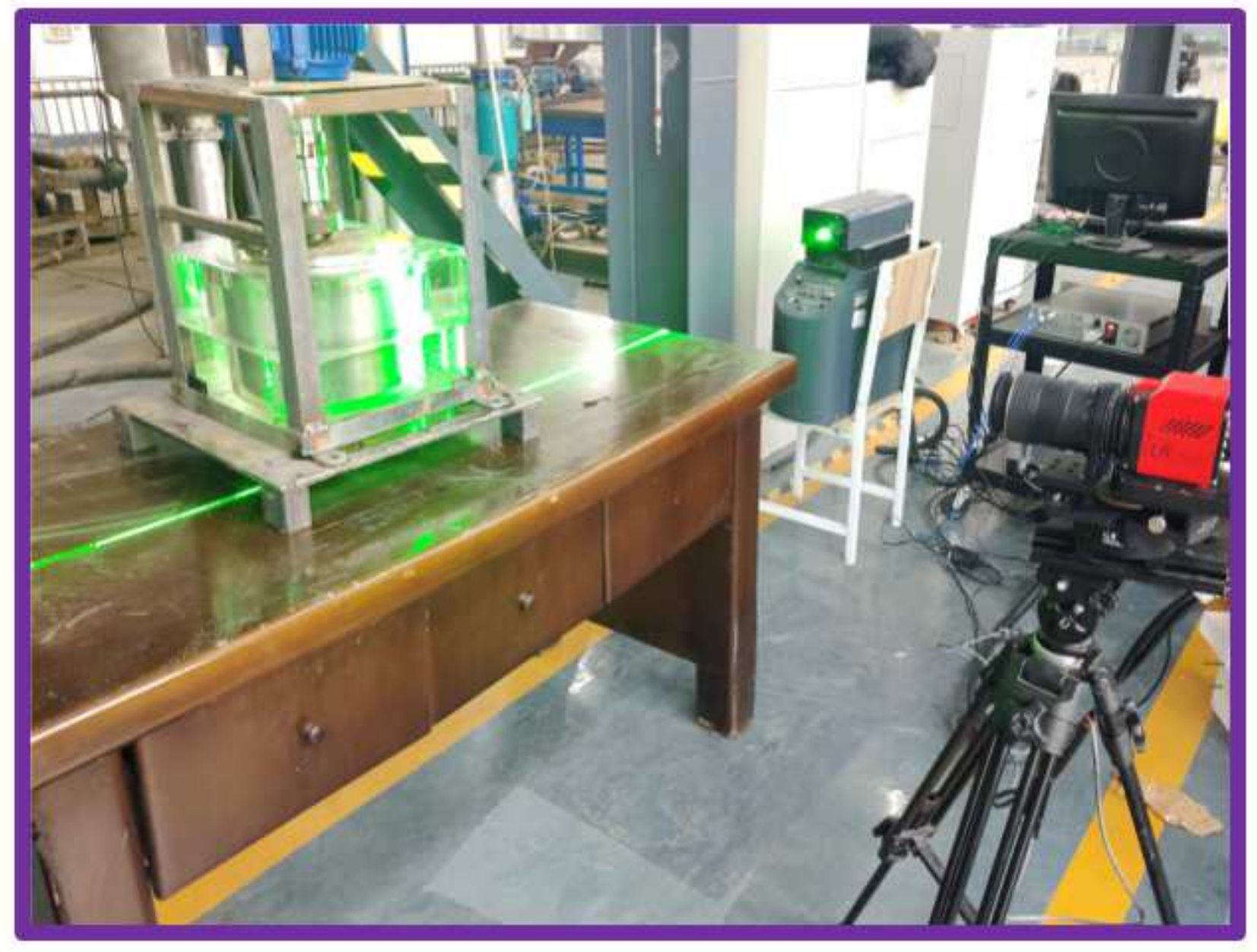

Figure 11

Field test set-up 


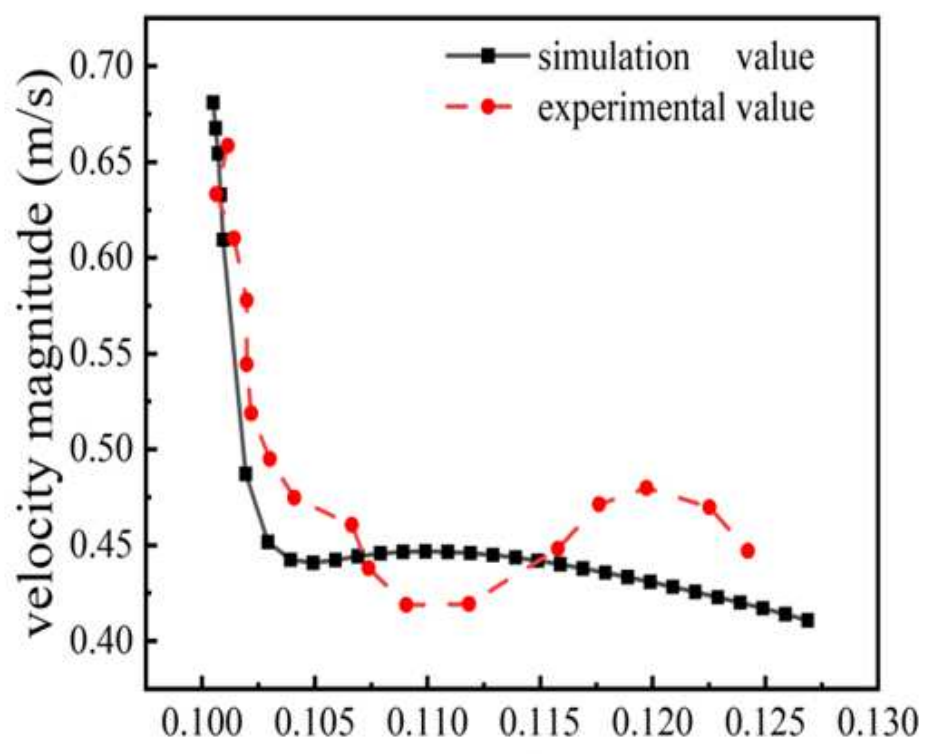

(a) Location's (m)

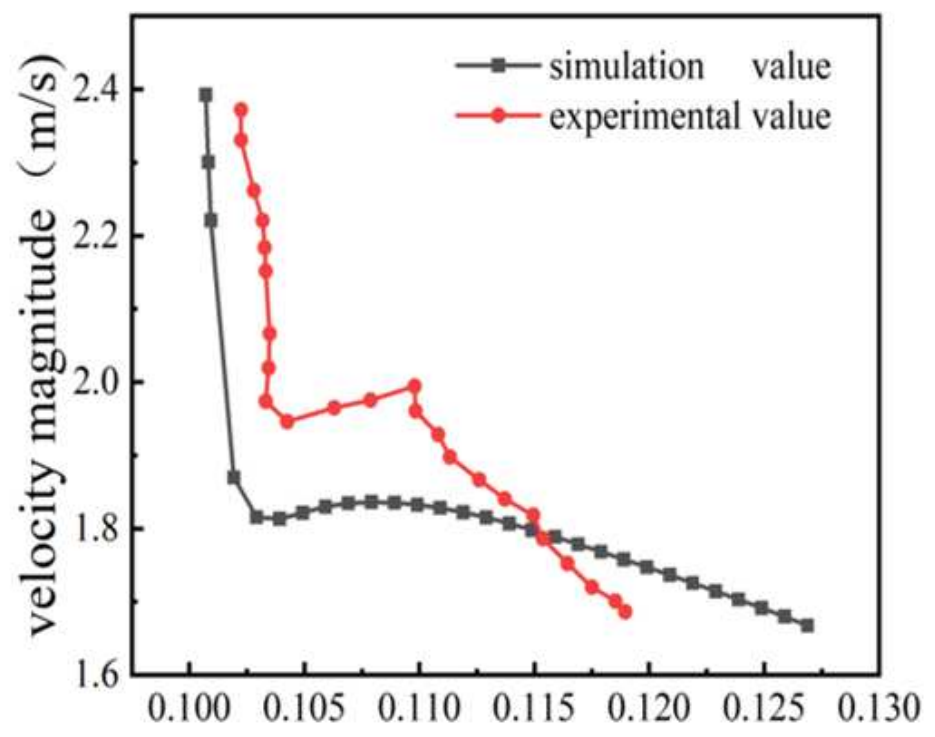

(c) Location's(m)

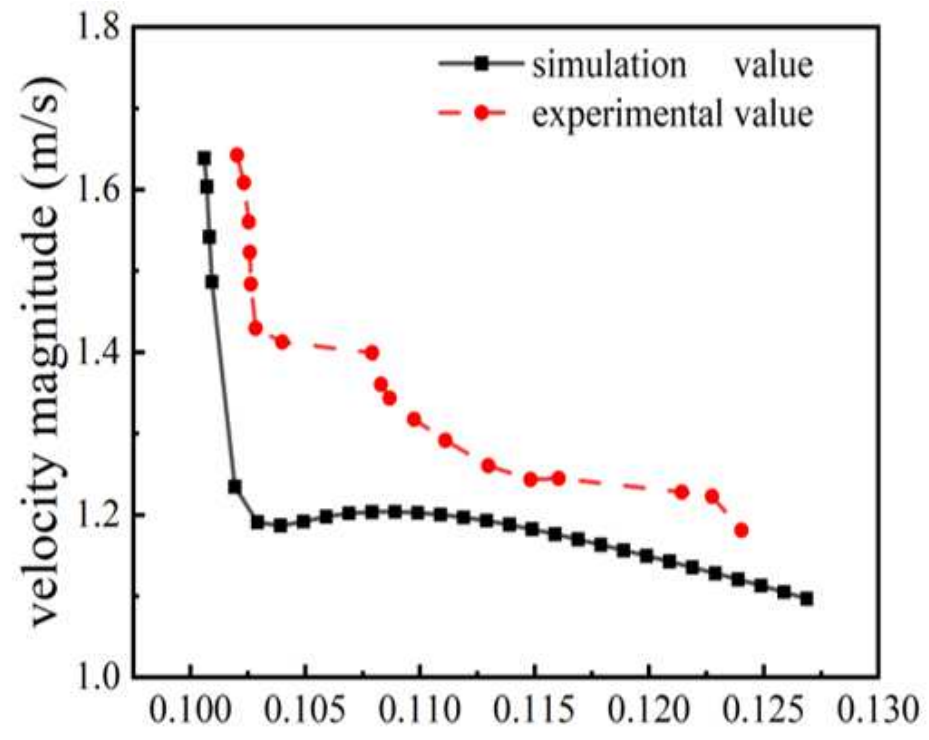

(b) Location's (m)

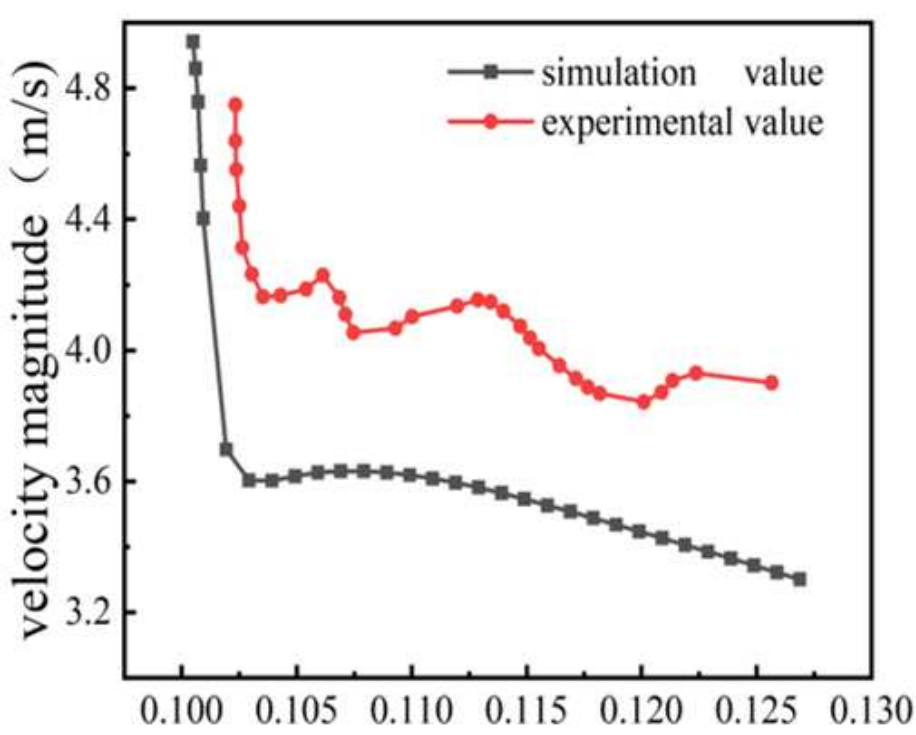

(d) Location's(m)

Figure 12

Comparison of experimental and simulation data ((a) $\omega=100 \mathrm{r} / \mathrm{min},(\mathrm{b}) \omega=300 \mathrm{r} / \mathrm{min}$, (c) $\omega=500$ $\mathrm{r} / \mathrm{min}$, and (d) $\omega=1000 \mathrm{r} / \mathrm{min}$ ) 